\title{
Spectrométrie instrumentale
}

\author{
P. Bouchareine \\ Institut d'Optique Théorique el Appliquèe, URA I4 du CNRS, Université Paris Sud, \\ B.P. 147, 91403 Orsay cedex. France
}

\begin{abstract}
Résumé : Étude instrumentale de la spectrométrie optique. Les définitions, les grandeurs mises en jeu sont passées en revue dans les deux premiers chapitres. Le troisiême chapitre étudie les instruments à fentes et leurs limitations. Le cas des spectromètres à prisme est étudié dans le quatrième chapitre et celui des spectromètres à réscaux dans le cinquième chapitre. La spectrométrie interférentielle est abordée avec l'interféromètre et le spectromètre de Fabry et Perol dans le sixième chapitre. Le septième chapitre décrit les principes de la spectrométrie de Fourier. Dans chaque chapitre on établit les relations entre la quantité de lumière acceptée par un spectromètre, définie par son étendue de faisceau, et le pouvoir de résolution que l'instrument permet d'atteindre. Dans le huitième et dernier chapitre, on montre que le pouvoir de résolution théorique de tous les spectromètres optiques peut se résumer en une formule quj traduit le caractère ondulatoire de la lumière, indépendamment des techniques expérimentales mises en oeuvre pour analyser la distribution spectrale de l'énergie dans un fajsceau de lumière.
\end{abstract}

1. SPECTROMÉTRIE OPTIQUE, TERMINOLOGIE, UNITÉS, DOMAINES SPECTRAUX

\subsection{Spectroscopes, spectromètres et spectrographes}

Un spectroscope est un instrument visuel, un spectromètre délivre le signal spectroscopique sous forme de signal photométrique, c'est la plupart du temps un appareil séquentiel avec lequel on balaye les éléments spectraux l'un après l'autre, mais il existe des spectromètres multicanaux qui analysent plusieurs éléments spectraux en parallèle et des spectromètres multiplexes qui analysent plusieurs éléments spectraux en même temps sur un même canal photométrique. Le terme spectrographe était réservé aux instruments utilisant le détecteur photographique, mais on l'applique plus généralement aux instruments qui utilisent un détecteur d'images (caméra électronique, détecteur matriciel). Nous emploierons uniformément le terme spectromètre pour désigner l'un ou l'autre type d'appareil spectrométrique. 


\subsection{Paramètres spectrométriques}

Longueurs d'onde $(\lambda$, mesurée en micromètres $(\mu \mathrm{m})$, nanomètres $(\mathrm{nm})$, picomètres $(\mathrm{pm})$ ou femtomètres (fm); éviter les Angströms ( $\AA$ ) qui ne sont plus utiles, depuis que l'unité de longueur est définie par des radiations atomiques ou par la vitesse de la lumière).

Fréquences $(v$ mesurée en hertz $(\mathrm{Hz})$, gigahertz $(\mathrm{GHz})$ ou térahertz $(\mathrm{THz})$. Les fréquences, difficilement accessibles en spectrométrie optique, sont rarement utilisées, si ce n'est en spectrométrie hétérodyne.

Nombres d'ondes $\left(\sigma\right.$, mesurés en centimètres ${ }^{-1}\left(\mathrm{~cm}^{-1}\right)$, plus rarement en mètres ${ }^{-1}$ $\left(\mathrm{m}^{-1}\right)$ ). Leur usage est recommandé dans tous les dispositifs interférentiels, car ce paramètre est proportionnel à la fréquence, et à l'angle de phase observé dans un système d'interférences.

On parle rarement de fréquences en spectrométrie optique, mais plutôt de longueurs d'onde $\lambda$, ou de nombres d'ondes $\sigma$. La limite de résolution $\delta \lambda$ ou $\delta \sigma$ caractérise l'aptitude d'un spectromètre à séparer deux radiations voisines. On utilise souvent un nombre sans dimension d'autant plus grand que la résolution est meilleure, le pouvoir de résolution $\mathrm{R}=$ $\lambda / \delta \lambda=\sigma / \delta \sigma$. Les pouvoirs de résolution vont de quelques centaines pour de petits spectromètres portatifs à plusieurs centaines de milliers pour les grands spectromètres à réseaux et plusieurs millions pour les spectromètres interférentiels. La métrologie interférentielle des longueurs d'onde atteint des exactitudes relatives $\lambda / \delta \lambda$ supérieures à $10^{10}$, qui sont plus ou moins assimilables à des pouvoirs de résolution.

\begin{tabular}{|c|c|c|c|c|c|c|c|c|}
\hline$v$ & $310^{11} \mathrm{~Hz}$ & $310^{12} \mathrm{~Hz}$ & $310^{13} \mathrm{~Hz}$ & $310^{14}$ & $14 \mathrm{~Hz}$ & $310^{15} \mathrm{~Hz}$ & $310^{16} \mathrm{~Hz}$ & $310^{17} \mathrm{~Hz}$ \\
\hline \multirow[t]{2}{*}{$\lambda$} & $1 \mathrm{~mm}$ & $100 \mu \mathrm{m}$ & $10 \mu \mathrm{m}$ & $1 \mu \mathrm{m}$ & & $100 \mathrm{~nm}$ & $10 \mathrm{~nm}$ & $1 \mathrm{~nm}$ \\
\hline & UHF & \multicolumn{2}{|c|}{ IRL } & $\mathbb{R}$ & Vis.UV & UVL & XUV & \\
\hline
\end{tabular}

Figure 1.1 : Les grands domaines spectraux des rayonnements électro-magnétiques.

De part et d'autre du domaine visible $(0,4 \mu \mathrm{m}<\lambda<0,75 \mu \mathrm{m})$ on distingue vers les courtes longueurs d'onde l'ultraviolet proche (UV : $0,4 \mu \mathrm{m}>\lambda>0,28 \mu \mathrm{m}$ ), l'ultraviolet lointain (UVL : $0,28 \mu \mathrm{m}>\lambda>0,1 \mu \mathrm{m}$ ) et l'extrême ultraviolet (XUV: $100 \mathrm{~nm}>\lambda>1 \mathrm{~nm}$ ), et vers les grandes longueurs d'onde l'infrarouge proche (IR : $0,8 \mu \mathrm{m}<\lambda<3 \mu \mathrm{m}$ ), l'infrarouge moyen $(3 \mu \mathrm{m}<\lambda<15 \mu \mathrm{m}$ ) et l'infrarouge lointain (IRL : $15 \mu \mathrm{m}<\lambda<300 \mu \mathrm{m}$ ). Au-delà de l'extrême ultraviolet nous trouvons le domaine des rayons $\mathbf{X}$ mous, et en-deçà de l'infrarouge lointain c'est le domaine des hyperfréquences. Les limites de ces domaines sont floues et sujettes à variations en fonction des progrès de l'instrumentation.

L'objet de la spectrométrie optique est l'étude de la distribution des densités spectrales de luminance (luminances spectriques). On distingue les sources qui ont un spectre de raies monochromatiques, caractérisé par des luminances $\mathrm{L}_{1}, \mathrm{~L}_{2}, \mathrm{~L}_{3}$ etc. et les sources qui émettent un spectre continu caractérisé par une densité spectrale de luminance ou luminance spectrique $L_{\lambda}$ ou $L_{\sigma}$. Le flux transmis par un spectromètre est le produit d'une luminance par une étendue de faisceau $U$ dans le premier cas, c'est le produit d'une densité spectrale de 
luminance $\mathrm{L}_{\lambda}\left(\mathrm{L}_{\sigma}\right)$ multipliée par une largeur spectrale $\delta \lambda(\delta \sigma)$ et par une étendue de faisceau $\mathrm{U}$ dans le second cas.

Tableau 1.1 : Quelques radiations de référence en spectroscopie

\begin{tabular}{|c|c|c|c|c|}
\hline \multicolumn{5}{|c|}{ Vitesse de la lumière dans le vide : $\mathrm{c}=299792458 \mathrm{~m} / \mathrm{s}$. } \\
\hline Transition & Fréquence & Ecart type & longueur d'onde & $\begin{array}{c}\text { Ecart type } \\
\text { relatif }\end{array}$ \\
\hline \begin{tabular}{|c|} 
krypton 86 \\
transition $2 \mathrm{p}_{10} 5 \mathrm{~d}_{5}$
\end{tabular} & $494886516,5 \mathrm{MHz}$ & $0,6 \mathrm{MHz}$ & $605780210,2 \mathrm{fm}$ & $1,310^{-9}$ \\
\hline \begin{tabular}{|c|} 
méthane transition \\
$\mathrm{F}_{2}{ }_{2}{ }_{3} \mathrm{P}(7)$
\end{tabular} & $\begin{array}{c}88376 \underset{\mathrm{kHz}}{181} 600,18 \\
\text { chen }\end{array}$ & $0,27 \mathrm{kHz}$ & $\begin{array}{c}3392231397,327 \\
\mathrm{fm}\end{array}$ & $310^{-12}$ \\
\hline \begin{tabular}{|c|} 
calcium 40 \\
transition ${ }^{3} P_{1}-{ }^{3} S_{0}$
\end{tabular} & $455986240,5 \mathrm{MHz}$ & $0,21 \mathrm{MHz}$ & $657459439,3 \mathrm{fm}$ & $4,510^{-10}$ \\
\hline \multicolumn{5}{|c|}{ Composantes hyperfines de transitions de l'iode 127.} \\
\hline $11-15 ; \mathrm{R}(127), \mathrm{i}$ & $473612214705 \mathrm{kHz}$ & $12 \mathrm{kHz}$ & $632991398,22 \mathrm{fm}$ & $2,510-11$ \\
\hline $9-2 ; \mathrm{R}(47), \mathrm{o}$ & $489880354,9 \mathrm{MHz}$ & $0,15 \mathrm{MHz}$ & $611970770,0 \mathrm{fm}$ & $310^{-10}$ \\
\hline $17-1 ; P(62), 0$ & $520206808,4 \mathrm{MHz}$ & $0,21 \mathrm{MHz}$ & $576294760,4 \mathrm{fm}$ & $410^{-10}$ \\
\hline $43-0 ; P(13), a_{3}(s)$ & $582490603,37 \mathrm{MHz}$ & $0,15 \mathrm{MHz}$ & $514673466,4 \mathrm{fm}$ & $2,510^{-10}$ \\
\hline
\end{tabular}

L'ouverture numérique des spectromètres est en général faible, on se trouve dans l'approximation de Gauss et l'étendue de faisceau peut s'écrire simplement par le produit d'une section droite $S$ et d'un angle solide $\Omega$, ou par le produit de sections droites $S$ et $S^{\prime}$ divisé par le carré de leur distance $\mathrm{D}$.

$$
\mathrm{U} \approx \mathrm{S} . \Omega \approx \frac{\mathrm{S} \cdot \mathrm{S}^{\prime}}{\mathrm{D}^{2}}
$$

L'angle solide d'un cône de révolution de demi-angle au sommet $i$ est rigoureusement égal à

$$
\Omega=2 \pi(1-\cos i)
$$

L'étendue de faisceau délimitée par une surface $\mathrm{S}$ éclairée sous l'angle solide ci-dessus est calculable exactement :

$$
\mathrm{U}=\pi \mathrm{S} \sin ^{2} \mathrm{i}
$$


Pour de faibles valeurs de $i$, l'approximation de Gauss nous ramène à $U=S . S^{\prime} / D^{2}$. Le tableau ci-après donne pour quelques valeurs de l'ouverture numérique le demi-angle en radians, l'angle solide exact en stéradians, l'étendue exacte et sa valeur approchée dans l'approximation de Gauss.

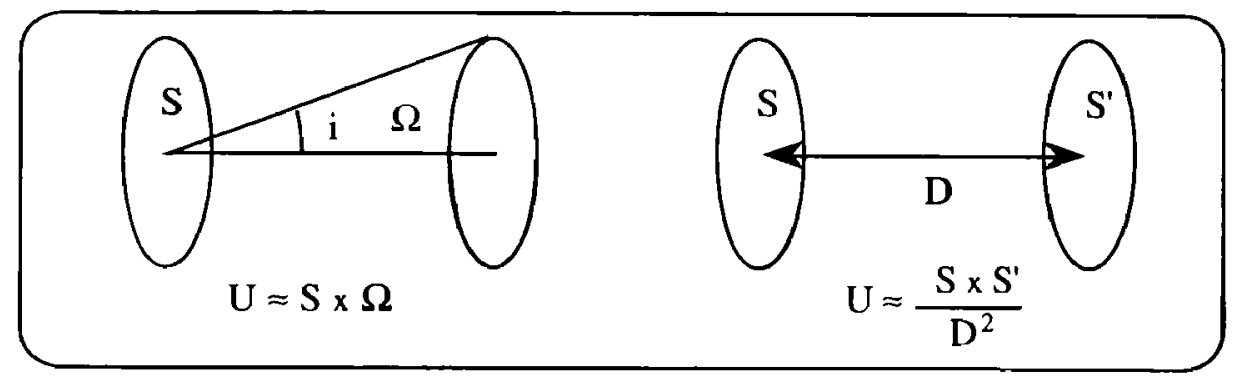

Figure 1.2 : Étendue de faisceau et angle solide : l'approximation de Gauss

$\mathrm{U} \approx \pi \mathrm{S} \mathrm{i^{2 }} \mathrm{sr}$, i étant exprimé en radians.

\begin{tabular}{lcccc} 
ouverture & $\begin{array}{c}\text { demi- } \\
\text { angle }\end{array}$ & angle solide & \multicolumn{1}{c}{ étendue } & $\begin{array}{c}\text { approximation } \\
\mathrm{S} \times \mathrm{S}^{\prime} / \mathrm{D}^{2}\end{array}$ \\
$\mathrm{f} / 50$ & $0,01 \mathrm{rad}$ & $3,14.10^{-4} \mathrm{sr}$ & $\mathrm{S} \times 3,14.10^{-4} \mathrm{~cm}^{2} . \mathrm{sr}$ & $\mathrm{S} \times 3,1410^{-4} \mathrm{~cm}^{2} . \mathrm{sr}$ \\
$\mathrm{f} / 25$ & $0,02 \mathrm{rad}$ & $1,26.10^{-3} \mathrm{sr}$ & $\mathrm{S} \times 1,26.10^{-3} \mathrm{~cm}^{2} . \mathrm{sr}$ & $\mathrm{S} \times 1,2610^{-3} \mathrm{~cm}^{2} . \mathrm{sr}$ \\
$\mathrm{f} / 10$ & $0,05 \mathrm{rad}$ & $7,8410^{-3} \mathrm{sr}$ & $\mathrm{S} \times 7,8310^{-3} \mathrm{~cm}^{2} . \mathrm{sr}$ & $\mathrm{S} \times 7,8510^{-3} \mathrm{~cm}^{2} . \mathrm{sr}$ \\
$\mathrm{f} / 5$ & $0,10 \mathrm{rad}$ & $3,1210^{-2} \mathrm{sr}$ & $\mathrm{S} \times 3,1110^{-2} \mathrm{~cm}^{2} . \mathrm{sr}$ & $\mathrm{S} \times 3,1410^{-2} \mathrm{~cm}^{2} . \mathrm{sr}$ \\
$\mathrm{f} / 2,5$ & $0,20 \mathrm{rad}$ & $1,2210^{-1} \mathrm{sr}$ & $\mathrm{S} \times 1,2110^{-1} \mathrm{~cm}^{2} . \mathrm{sr}$ & $\mathrm{S} \times 1,2610^{-1} \mathrm{~cm}^{2} . \mathrm{sr}$ \\
$\mathrm{f} / 1$ & $0,46 \mathrm{rad}$ & $0,66 \mathrm{sr}$ & $\mathrm{S} \times 0,63 \mathrm{~cm}^{2} . \mathrm{sr}$ & $\mathrm{S} \times 0,79 \mathrm{~cm}^{2} . \mathrm{sr}$
\end{tabular}

\section{CARACTÉRISATION D’UN SPECTROMÈTRE}

Fonction d'appareil, étendue de faisceau et luminosité, variations avec le pouvoir de résolution

\subsection{Fonction d'appareil}

Un spectromètre éclairé par une radiation parfaitement monochromatique de longueur d'onde $\lambda_{0}$ et de luminance $L_{o}$ donne une réponse $L_{0} A\left(\lambda-\lambda_{0}\right)$. Si la réponse du spectromètre est stationnaire, la fonction $A(\lambda)$ est indépendante de $\lambda_{0}$, elle est appelée la fonction d'appareil. Si le spectromètre est linéaire, sa réponse à une densité spectrale quelconque $\partial L / \partial \lambda$ s'écrit :

$$
R\left(\lambda_{0}\right)=\int L_{\lambda}(\lambda) A\left(\lambda_{0}-\lambda\right) d \lambda
$$

La largeur à mi-hauteur de la fonction $A(\lambda)$ est la limite de résolution $\delta \lambda d u$ spectromètre. La hauteur de la fonction $A(\lambda)$ est l'étendue de faisceau du spectromètre et caractérise sa luminosité, c'est-à-dire son aptitude à transmettre un flux donné pour une source donnée. 
Le flux transmis par un spectromètre éclairé par une radiation monochromatique de luminance $\mathrm{L}_{\mathrm{o}}$ s'écrit :

$$
\mathbf{F}=\mathrm{L}_{\mathrm{o}} \mathrm{U}
$$
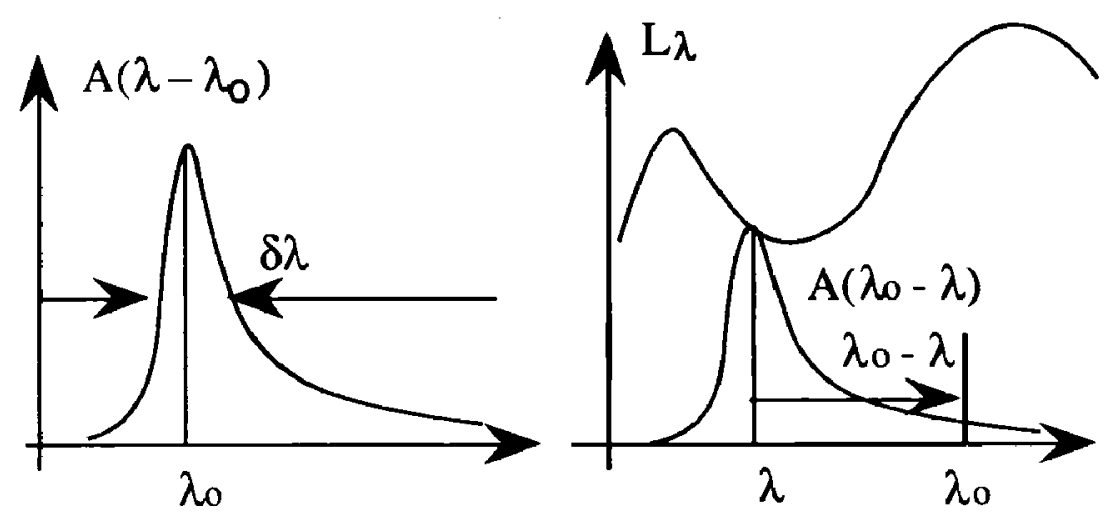

Figure 2.1 : La fonction d'appareil et son action sur un spectre.

Ce flux est proportionnel à la hauteur de la fonction d'appareil, c'est-à-dire à l'étendue de faisceau U acceptée par le spectromètre.

Le flux transmis par un spectromètre éclairé par une source dont la luminance spectrique est constante (source blanche, notion valable sur un intervalle spectral étroit) s'écrit :

$$
F=L_{\lambda} \int A(\lambda) \delta \lambda \approx L_{\lambda} U \delta \lambda
$$

Ce flux est proportionnel à la surface de la fonction d'appareil, c'est-à-dire en première approximation au produit de sa hauteur $U$ par sa largeur à mi-hauteur $\delta \lambda$.

Exemple : Une source émet une radiation monochromatique de luminance $\mathrm{L}_{\mathrm{o}}$ superposće à un fond continu de densité spectrale de luminance $L_{\lambda}$. Donner les réponses d'un spectromètre avec deux fonctions d'appareil de forme triangulaire ayant même hauteur $\mathrm{U}=1 \mathrm{~cm}^{2} . \mathrm{sr}$ et les largeurs :

$$
\delta \lambda_{1}=0,1 \mathrm{~nm} \text { et } \delta \lambda_{2}=10 \mathrm{~nm} .
$$

Application numérique : $\mathrm{L}_{\mathrm{o}}=0,1 \mathrm{~W} / \mathrm{cm}^{2} . \mathrm{sr}$

$$
\begin{array}{lll}
\left.1^{\circ}\right) \delta \lambda=10 \mathrm{~nm} & \Phi_{0}=\mathrm{L}_{0} \mathrm{U}=0,1 \mathrm{~W} & \mathrm{~L}_{\lambda}=0,1 \mathrm{~W} / \mathrm{cm}^{2} . \mathrm{sr} . \mathrm{nm} \\
\left.2^{\circ}\right) \delta \lambda=0,1 \mathrm{~nm} & \Phi_{0}=0,1 \mathrm{~W} & \Phi_{\lambda}=\mathrm{L} \lambda \mathrm{U} \delta \lambda=1 \mathrm{~W}
\end{array}
$$

On voit que le rapport des flux monochromatique $\Phi_{0}$ et continu $\Phi_{\lambda}$ dépend du pouvoir de résolution mais ne saurait être une caractéristique intrinsèque de la source. 


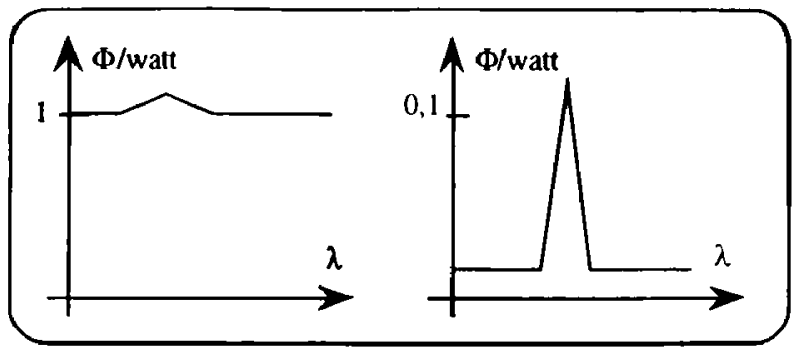

Figure 2.2: Le spectre d'une même source vu par deux spectromètres différents.

\section{LES SPECTROMÈTRES À DISPERSEUR ET FENTES}

Pouvoir de résolution théorique, relation entre l'étendue de faisceau et le pouvoir de résolution, adaptation d'étendue avec un instrument : emploi des fibres optiques et des dissecteurs d'images.

\subsection{Relation de base}

Un disperseur est caractérisé par une relation fondamentale entre angle d'incidence $i_{1}$, angle d'émergence i2 et longueur d'onde $\lambda$ :

$$
f\left(i_{1}, i_{2}, \lambda\right)=0
$$

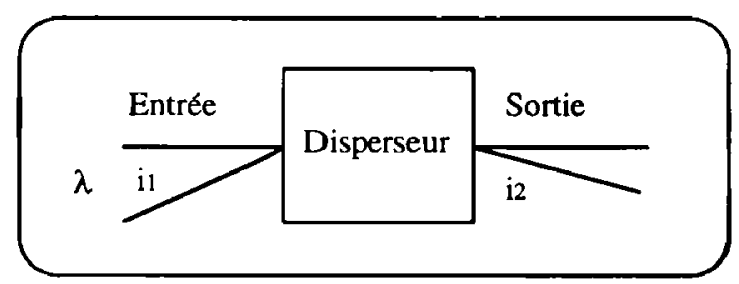

Figure 3-1 : les paranètres d'un spectromètre dispersif

De cette relation nous tirons les paramètres caractéristiques d'un spectromètre à disperseur qui sont les dispersions d'entrée $D_{1}=\partial \mathrm{i}_{1} / \partial \lambda$ et de sortie $D_{2}=\partial \mathrm{i}_{2} / \partial \lambda$, le grossissement angulaire $\mathrm{G}=\partial \mathrm{i}_{2} / \partial \mathrm{i}_{1}=\mathrm{D}_{2} / \mathrm{D}_{1}$ et le grandissement pupillaire qui lui est lić $\gamma=1 / G$.

Les fentes d'un spectromètre sont caractérisées par leur largeur angulaire ( $\alpha_{1}$ à l'entrée, $\alpha_{2}$ à la sortie) à laquelle correspond une largeur spectrale $\delta \lambda_{1}=\alpha_{1} \mathcal{D}_{1}, \delta \lambda_{2}=\alpha_{2} \mathcal{D}_{2}$. On montre que la meilleure fonction d'appareil est obtenue lorsque la largeur angulaire de la fente de sortie est égale à la largeur angulaire de l'image de la fente d'entrée. Les largeurs spectrales des fentes sont alors égales; la fonction d'appareil a une forme triangulaire et sa largeur à mihauteur est égale à la largeur spectrale commune aux deux fentes. 


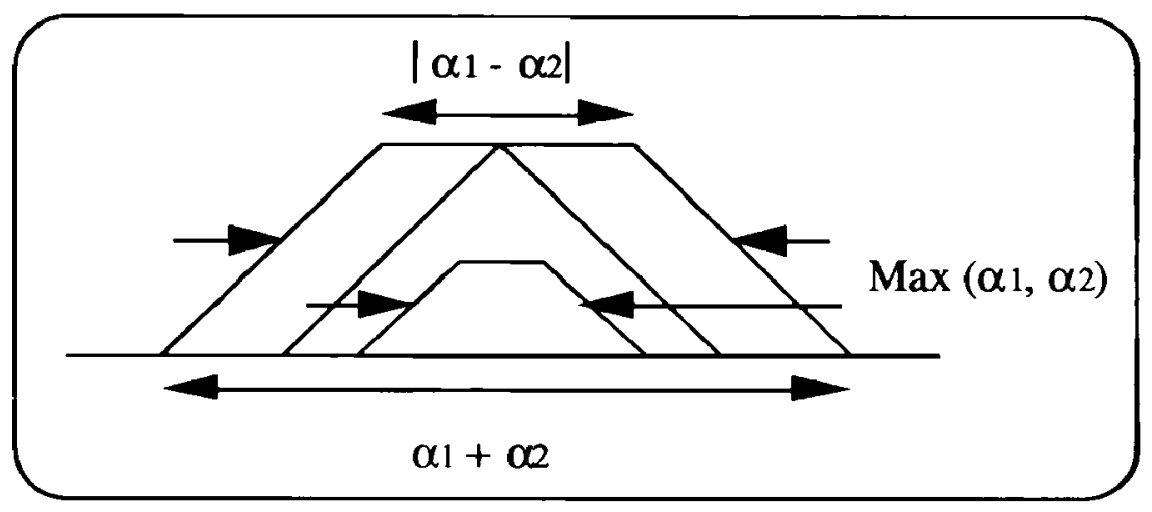

Figure 3.2: Fonctions d'appareil d'un spectromètre à fentes en fonction de $\alpha_{1}$, largeur angulaire de l'image de la fente d'entréc et de $\alpha_{2}$ largeur angulaire de la fente de sortie ( $\alpha_{2}$ constant, $\alpha_{1}$ inférieur, égal et supérieur à $\alpha_{2}$ ).

\subsection{Diffraction de la lumière, pouvoir de résolution théorique}

La plus petite largeur angulaire que l'on puisse donner à la fente d'entrée est la limite de résolution de la pupille d'entrée déterminée par la diffraction. Si la pupille est rectangulaire de largeur $l_{1}$, la largeur angulaire de la tache de diffraction (à peu près la largeur à mi-hauteur) est $\lambda / 1_{1}$. Si la pupille est circulaire, cette largeur angulaire est égale à $1,22 \lambda \Lambda_{1}$. De même la plus petite largeur angulaire de la fente de sortie est la largeur de la tache de diffraction de la pupille de sortie. Il en résulte une valeur limite du pouvoir de résolution, appelé pouvoir de résolution théorique. On peut constater que la largeur correspondante de la pupille de sortie $l_{2}$ est telle que les largeurs spectrales limitées par la diffraction sont égales.

$$
\begin{gathered}
\alpha_{2}=|\mathrm{G}| \alpha_{1} \text { où } \mathrm{G}=\partial \mathrm{i}_{2} / \partial \mathrm{i}_{1}=-\mathrm{D}_{2} / \mathrm{D}_{1} . \quad \delta \lambda_{1}=\delta \lambda_{2}=\alpha / \mathrm{D} \\
\mathrm{R}=\lambda / \delta \lambda=\lambda \mathrm{D} / \alpha \\
\lambda /\left(\mathrm{l}_{1} \mathrm{D}_{1}\right)=\lambda /\left(\mathrm{l}_{2} \mathrm{D}_{2}\right)=\lambda /(\mathrm{D}) \\
\mathrm{R}_{\mathrm{o}}=\lambda \mathrm{D} / \alpha_{\min }=1 \mathrm{D}=1_{1} \mathrm{D}_{1}=\mathrm{l}_{2} \mathrm{D}_{2} .
\end{gathered}
$$

Le pouvoir de résolution théorique ne peut pas être atteint avec un spectromètre à fentes. Il faudrait en effet pour cela que les largeurs angulaires des fentes d'entrée et de sortie soient très petites devant les largeurs des taches de diffraction, et la luminosité serait catastrophique. Le pouvoir de résolution théorique n'est qu'une limite asymptotique avec les spectromètres à fentes.

Un spectromètre à fentes dont les largeurs spectrales des fentes sont égales offre un pouvoir de résolution

$$
\mathbf{R}=\lambda \mathrm{D} / \alpha
$$




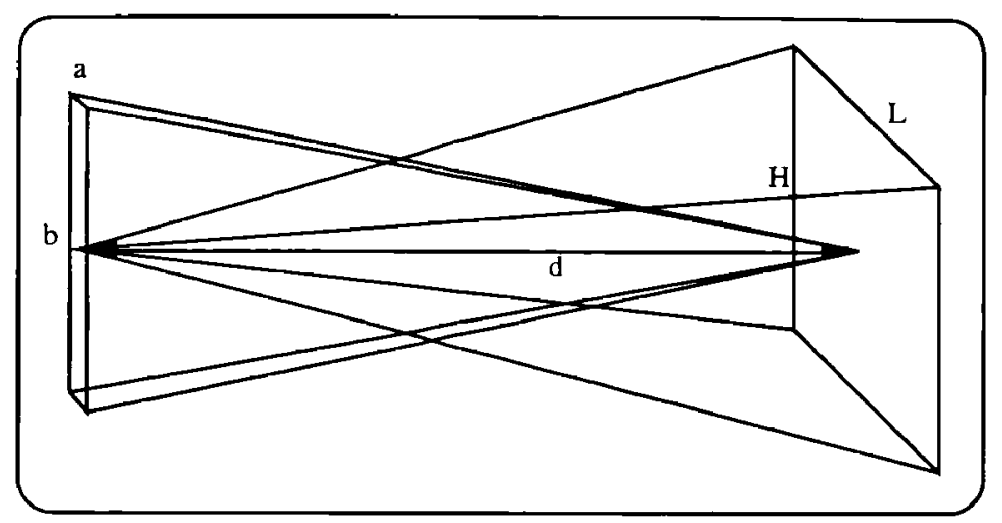

Figure 3.3 : L'étendue de faisceau acceptée par un spectromètre à fentes.

La largeur angulaire des fentes est $\alpha$, leur hauteur angulaire est $\beta$. L'étendue de faisceau disponible $\mathrm{U}$ est égale au produit de la surface de la section droite $\mathrm{S}$ du disperseur par l'angle solide $\Omega=\alpha \beta$ déterminé par les fentes. La surface $S$ de la section droite du disperseur est le produit de sa hauteur $\mathrm{H}$ par la largeur I. Le produit de l'étendue de faisceau U par le pouvoir de résolution $\mathbf{R}$ s'écrit

$$
\mathrm{U} . \mathrm{R}=\lambda \mathrm{D} / \alpha \mathrm{H} \mathrm{l} \alpha \beta=\lambda \beta \mathrm{HR} \mathrm{R}_{\mathbf{O}}
$$

qui est à peu près une constante pour un instrument déterminé. En effet nous verrons que le pouvoir de résolution théorique $\mathrm{R}_{\mathrm{o}}$ des appareils à disperseur est à peu près proportionnel à l'inverse de la longueur d'onde $\lambda$.

Une difficulté spécifique des instruments à fentes est la grande disproportion entre la hauteur angulaire et la largeur angulaire des fentes. Il est possible de s'adapter à cette géométrie anamorphosée avec des dispositifs optiques complexes appelés dissecteurs d'image. Une réalisation plus simple est possible grâce aux fibres optiques. Dissecteurs d'images et faisceaux de fibres optiques permettent d'adapter la géométrie d'une étendue de faisceau à celle d'un spectromètre à fentes. On peut ainsi transformer une étendue de faisceau horizontale en étendue de faisceau verticale, mais il ne sera jamais possible de réduire l'étendue d'un faisceau sans perdre de lumière pour le faire entrer dans un spectromètre, car cela augmenterait sa luminance, ce qui serait contraire au deuxième principe de la thermodynamique.

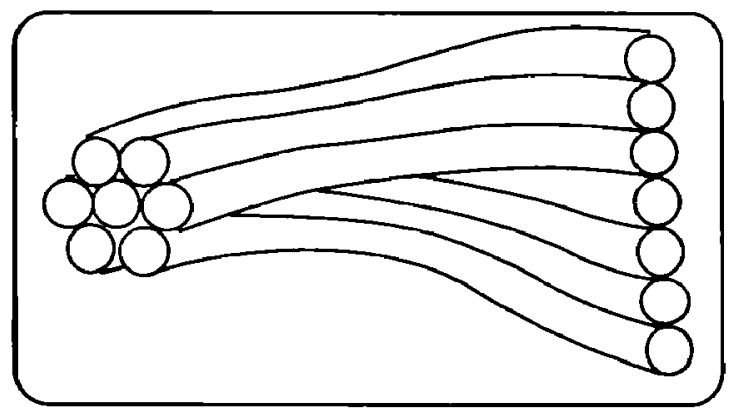

Figure 3.4 : Un dissecteur d'image à fibres optiques (d'après document de l'Observatoire de Paris-Meudon). 


\section{SPECTROMÈTRE À PRISME}

Formules fondamentales, dispersion, grandissement, grossissement, limite et pouvoir de résolution, pouvoir de résolution théorique, dispositifs expérimentaux.

\subsection{Relations de base}

Les relations de Descartes permettent d'établir les variations de $i_{1}$ et $i_{2}$ en fonction de la longueur d'onde $\lambda$.

$$
\begin{gathered}
\sin i_{1}=n \sin r_{1} \\
r_{1}+r_{2}=A \\
\sin i_{2}=n \sin r_{2}
\end{gathered}
$$

Par convention les angles $i_{1}$ et $i_{2}$ sont toujours comptés positifs, bien qu'ils soient de signes contraires à l'entrée et à la sortie, il en est de même pour $r_{1}$ et $r_{2}$. Dans l'expression du grossissement angulaire $\partial \mathrm{i}_{2} / \partial \mathrm{i}_{1}$, il faut s'en souvenir pour retrouver que $\mathrm{i}_{2}$ augmente quand $\mathrm{i}_{1}$ diminue.

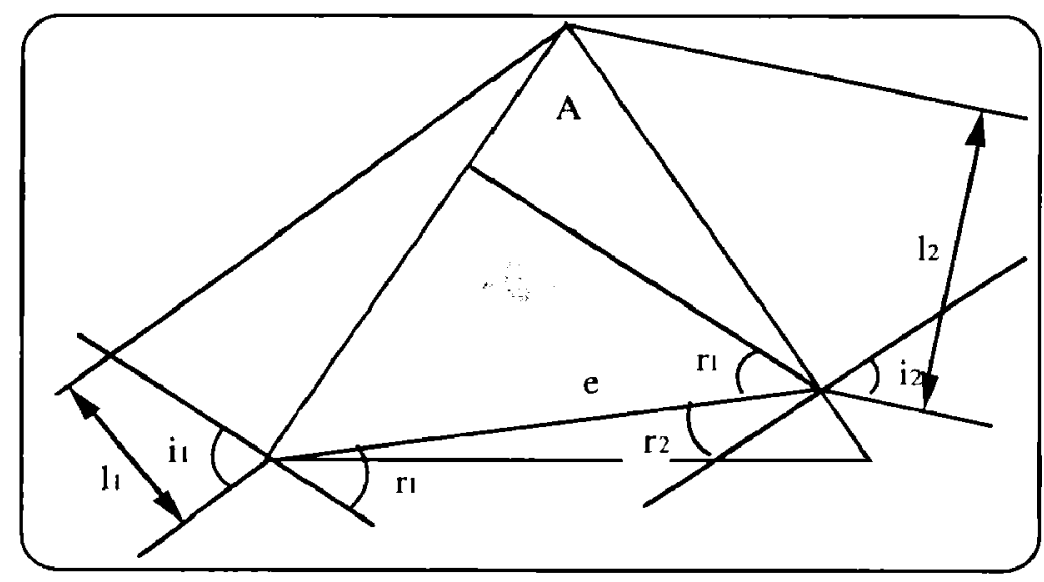

Figure 4.1: Les paramètres d'un spectromètre à prisme

La dispersion $\mathrm{D}_{2}$ d'un spectromètre à prisme s'écrit :

$$
D_{2}=\frac{\sin A}{\cos r_{1} \cos i_{2}} \frac{\partial n}{\partial \lambda}
$$

Par raison de symétrie, la dispersion $\mathrm{D}_{1}$ s'écrit :

$$
D_{1}=\frac{\sin A}{\cos r_{2} \cos i_{1}} \frac{\partial n}{\partial \lambda}
$$


et le grossissement angulaire s'écrit

$$
G=\frac{\cos r_{2} \cos i_{1}}{\cos r_{1} \cos i_{2}}
$$

Les largeurs $l_{1}$ et $l_{2}$ des pupilles d'entrée et de sortie s'expriment en fonction de l'épaisseur de verre e à la base du faisceau par

$$
l_{1}=\frac{e \cos r_{2} \cos i_{1}}{\sin A} \quad l_{2}=\frac{e \cos r_{1} \cos i_{2}}{\sin A}
$$

d'où l'on tire l'expression du pouvoir de résolution théorique $\mathbf{R}_{0}$ :

$$
\mathrm{R}_{0}=\mathrm{e} \frac{\partial \mathrm{n}}{\partial \lambda}=\frac{\mathrm{e}}{\lambda} \lambda \frac{\partial \mathrm{n}}{\partial \lambda}
$$

Il est difficile de fabriquer un prisme bien homogène dont la base dépasse une dizaine de centimètres. La quantité sans dimension $\lambda \partial n / \partial \lambda$ est imposée par la nature et varie de quelques $10^{-3}$ pour la silice dans le rouge à quelques $10^{-2}$ pour un flint lourd dans le violet. Les pouvoirs de résolution correspondant ne dépassent pas quelques milliers dans le domaine visible.

\subsection{Réalisations pratiques}

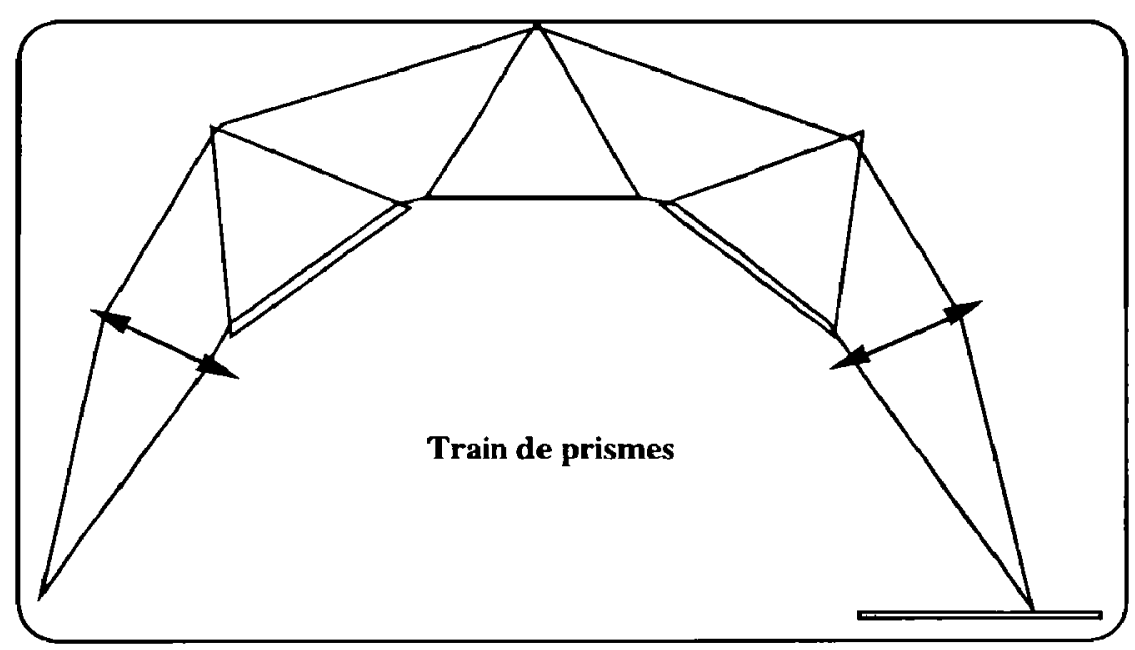

Figure 4.2 : Spectrographe à train de prismes.

On peut mettre plusieurs prismes en série (jusqu'à trois ou quatre) pour augmenter le pou voir de résolution d'un spectromètre à prismes (fig. 4.2). 
On peut aussi compenser la déviation en mettant en série des prismes montés tête-bêche dans un montage à vision directe connu sous le nom de montage d'Ámici (fig. 4.3).

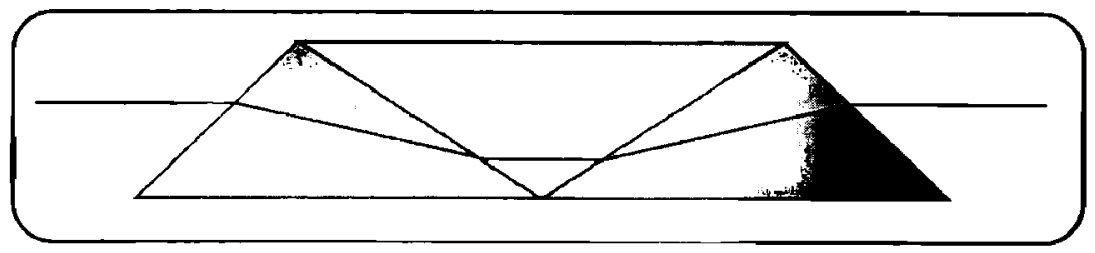

Figure 4.3: Montage d'Amici à vision directe

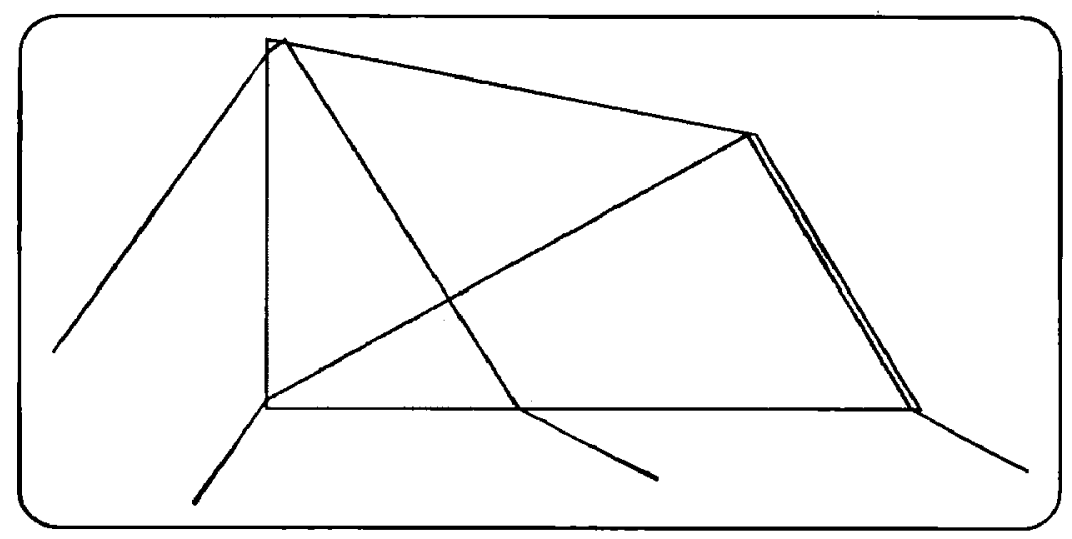

Figure 4.4 : Prisme de Pellin-Broca à déviation rectangulaire.

Le prisme de Pellin-Broca est un prisme à réflexion interne qui garantit une déviation constante égale à $90^{\circ}$ pour tout faisceau traversant le prisme au minimum de déviation. Ce prisme a équipé de nombreux monochromateurs largement utilisés dans les laboratoires (fig. 4.4).

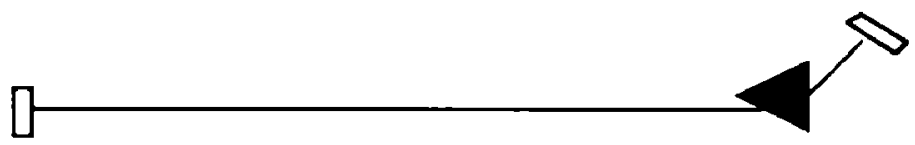

Figure 4.5: Prisme sélecteur dans une cavité laser

Un prisme placé à l'intérieur d'un cavité laser est traversé un grand nombre de fois par la lumière. Son pouvoir de résolution est augmenté d'autant. S'il est utilisé sous l'incidence de Brewster, les pertes pour une onde polarisée avec son champ électrique dans le plan d'incidence (plan de la figure 4.5 ) sont très faibles.

On peut aussi mettre dans la cavité d'un laser à colorant un train de prismes en compensant leurs dispersions et en multipliant leurs grandissements pupillaires pour adapter 
la faible largeur du milieu amplificateur (quelques millimètres) à la largeur d'un réseau sélecteur de fréquence dans un laser à longueur d'onde accordable (fig. 4.6).

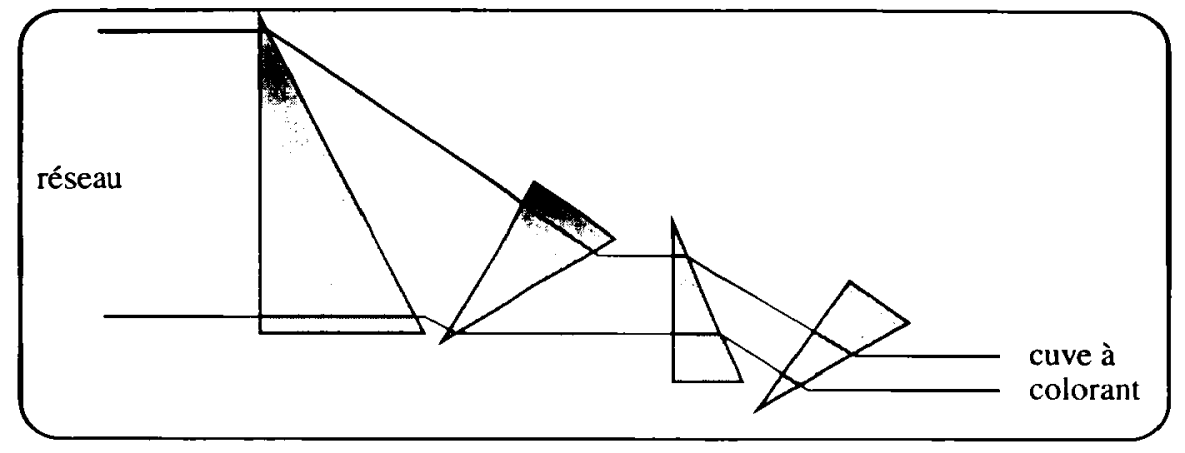

Figure 4.6 : Train de prismes anamorphoseurs dans une cavité laser.

\subsection{Courbure de l'image de fente donnée par un prisme}

La fente à l'infini envoie sur le prisme des rayons qui ne sont pas tous dans le plan de section principale. Si la hauteur angulaire de la fente est égale à $\beta$, l'angle d'incidence maximal des rayons avec le plan de section principale est égal à $\beta / 2$. Les relations entre les angles $i_{\mid}$et $i_{2}$ ne sont pas les mêmes pour ces rayons et pour les rayons du plan de section principale pour lesquels nous avons fait les calculs.

Pour établir ces relations, il faut écrire les lois de Descartes dans l'espace à trois dimensions, et le problème est compliqué par le fait que la trajectoire d'un rayon au cours des réfractions sur les deux faces du prisme n'est plus contenue dans un plan. On peut cependant faire une théorie simplifiée de la réfraction en projetant les rayons dans un plan de section principale. On trouve alors que les relations de Descartes sont valables pour ces projections avec une valeur modifiée $n$ ' de l'indice qui dépend de $\beta$ et que l'on appelle l'indice projeté :

$$
\mathrm{n}^{\prime}=\mathrm{n}+\frac{\beta^{2}}{8} \frac{\mathrm{n}^{2}-1}{\mathrm{n}}
$$

Il résulte de cette expression que l'image d'une fente rectiligne à l'infini donnée par un prisme est un arc de cercle dont la flèche de courbure exprimée en longueur d'onde est :

$$
\delta \lambda=\frac{\delta n}{\operatorname{dn} / d \lambda}=\frac{\beta^{2}}{8} \frac{n^{2}-1}{n} \frac{1}{d n / d \lambda}
$$

Pour $\mathrm{n}=1,618, \frac{n^{2}-1}{n}=1$. Si l'on veut que la flèche n'excède pas la largeur spectrale des fentes $\delta \lambda$, la hauteur angulaire des fentes $\beta$ doit être limitée en fonction du pouvoir de résolution $\mathrm{R}$ :

$$
\beta<2 \sqrt{\frac{2 \lambda \operatorname{dn} / \mathrm{d} \lambda}{R}}
$$


Application numérique : $R=1000, \lambda \partial \mathrm{n} / \partial \lambda=10^{-2}, \beta<9 \mathrm{mrad}$.

\section{SPECTROMÈTRE A RÉSEAU}

Relation de base, dispersion, grandissement, grossissement, limite et pouvoir de résolution, pouvoir de résolution théorique, la multiplicité et la superposition des ordres, montages expérimentaux.

\subsection{Relation de base}

La relation générale des réseaux s'écrit :

$$
a\left(\sin i_{1}+\sin i_{2}\right)=k \lambda
$$

où $i_{1}$ est l'angle du rayon incident avec la normale au réseau, $i_{2}$ l'angle du rayon émergent. Ces angles sont algébriques. Ils seront orientés de la même manière pour un réseau par réflexion, mais pour conserver la même relation avec un réseau par transmission, nous orienterons les angles dans deux sens opposés de part et d'autre du réseau.

L'ordre zéro correspond ainsi à la réflexion spéculaire d'un miroir pour un réseau par réflexion et à la transmission rectiligne directe pour un réseau par transmission. Le signe des ordres est par contre arbitraire et n'a pas de signification physique.
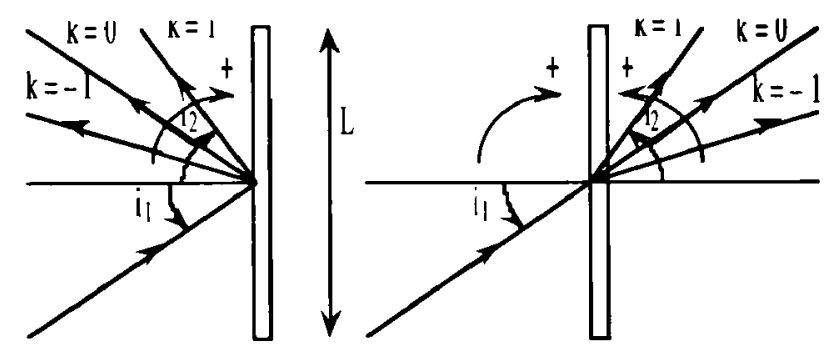

Figure 5.1 : les paramètres (ordre $k$, angles d'incidence et d'émergence) pour un réseau plan par réflexion (à gauche) et pour un réseau plan par transmission (à droite). Les sens positifs de mesure des angles sont conventionnels.

La dispersion d'un réseau s'obtient beaucoup plus simplement que celle d'un prisme :

$$
\begin{aligned}
& D_{1}=\frac{\partial i_{1}}{\partial \lambda}=\frac{k}{a \cos i_{1}} \\
& D_{2}=\frac{\partial i_{2}}{\partial \lambda}=\frac{k}{a \cos i_{2}}
\end{aligned}
$$

Le grossissement angulaire est $G=\frac{\cos i_{1}}{\cos i_{2}}$ et le grandissement pupillaire est $\gamma=\frac{\cos i_{2}}{\cos i_{1}}$. La largeur de la pupille de sortie est $\mathrm{I}_{2}=\mathrm{L} \cos \mathrm{i}_{2}$ 


$$
\text { d'où } R_{0}=l_{2} D_{2}=\frac{L . k}{a}=N . k
$$

Si $N$ est grand, $k$ est limité parce que $\sin i_{1}+\sin i_{2}$ reste inférieur à 2 . Si $k$ est élevé, $N$ est nécessairement réduit. On obtient une expression plus significative de $R_{0}$ en remplaçant $k$ par son expression déduite de la relation générale :

$$
R_{0}=\frac{L\left(\sin i_{1}+\sin i_{2}\right)}{\lambda}=\frac{D_{\max }}{\lambda}
$$

où l'on remarque que $L\left(\sin i_{1}+\sin i_{2}\right.$ ) représente la différence de marche maximale entre les rayons extrêmes reçus par le réseau. Un bon réseau peut atteindre 250 à $300 \mathrm{~mm}$ de largeur, avec des angles $i_{1}$ et $i_{2}$ allant de $30^{\circ}$ pour des réseaux ordinaires jusqu'à $75^{\circ}$ pour certains réseaux de haute dispersion. Les pouvoirs de résolution théoriques correspondants vont de quelques dizaines de milliers à plusieurs centaines de milliers.

Les réseaux atteignent des pouvoirs de résolution plus de cent fois plus grands que ceux des prismes. Pour des pouvoirs de résolution identiques, un spectromètre à réseau offre une étendue de faisceau plus de cent fois supérieure à celle d'un spectromètre à prisme.

$$
\mathrm{UR}=\lambda \beta \mathrm{HR}_{\mathrm{O}}
$$

Par ailleurs la relation entre les angles et les longueurs d'onde comporte avec un prisme une fonction non linéaire $\partial n / \partial \lambda$ que seule l'expérience permet d'approcher. La relation équivalente avec un réseau est exclusivement géométrique. Il suffit de mesurer des angles pour obtenir le rapport de deux longueurs d'onde, sans même avoir besoin de la mesure du pas a du réseau. La spectrométrie par réseau a fait faire d'énormes progrès à la mesure des longueurs d'onde avant les mesures interférométriques de Michelson et Fabry.

\subsection{Multiplicité et superposition des ordres}

Le réseau est un spectromètre interférentiel. Il est donc soumis au sérieux problème de la superposition des ordres. Si une longueur d'onde $\lambda_{k}$ est transmise pour une incidence $i_{1}$ et une émergence $i_{2}$ dans l'ordre $k$, les longueurs d'ondes suivantes sont également transmises dans les même directions dans les ordres $k-1$ et $k+1$ :

$$
a\left(\sin i_{1}+\sin i_{2}\right)=k \lambda_{k}=(k+1) \lambda_{k+1}=(k-1) \lambda_{k-1}
$$

S'agissant d'un dispositif interférentiel, il est intéressant de transformer ces relations sur les longueurs d'onde en relations sur les nombres d'ondes :

$$
a\left(\sin i_{1}+\sin i_{2}\right)=k / \sigma_{k}=(k+1) / \sigma_{k+1}=(k-1) / \sigma_{k-1}
$$

d'où l'on obtient

$$
\sigma_{\mathrm{k}} / \mathrm{k}=\sigma_{\mathrm{k}+1} /(\mathrm{k}+1)=\sigma_{\mathrm{k}-1} /(\mathrm{k}-1)=\sigma_{1}=\sigma_{\mathrm{k}+1}-\sigma_{\mathrm{k}}=\sigma_{\mathrm{k}}-\sigma_{\mathrm{k}-1}=\Delta \sigma
$$

Les nombres d'ondes transmis dans les différents ordres d'un réseau pour une même direction d'incidence $i_{1}$ et une même direction d'émergence $i_{2}$ sont tous multiples du nombre d'ondes $\sigma_{1}$ transmis dans le premier ordre. L'intervalle entre ordres $\Delta \sigma$ est constant. On l'appelle l'intervalle spectral libre et il est égal à $\sigma_{1}$. 
De cette étude il résulte que le problème de superposition des ordres n'est pas spécifique à un réseau particulier. Il est identique pour tous les réseaux. C'est un simple problème d'arithmétique lié à l'ordre dans lequel on travaille. Pour un intervalle spectral total étudié égal à une octave (domaine visible entre $\lambda=0,4 \mu \mathrm{m}$ et $\lambda=0,8 \mu \mathrm{m}$ ) la superposition des ordres est représentée pour les premiers ordres sur le schéma de la figure 5.2. Il n'y a jamais superposition des ordres dans le visible pour un réseau qui travaille dans le premier ordre, ni dans le deuxième ordre pour des radiations de longueur d'onde inférieure à $0,6 \mu \mathrm{m}$. On isole le deuxième et le troisième ordre avec de simples filtres colorés. Au-delà, il faut faire appel à un prémonochromateur dont le pouvoir de résolution $R=\sigma / \Delta \sigma$ est égal à $k$, l'ordre dans lequel on observe la radiation de nombre d'ondes $\sigma$.

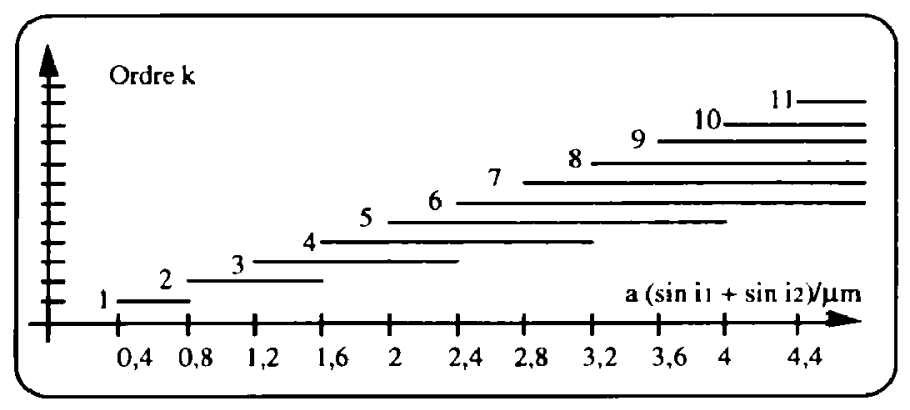

Figure 5.2 : La superposition des ordres dans le domaine visible

\subsection{Courbure de fente dans un spectromètre à réseau.}

Le calcul est beaucoup plus simple que pour le prisme, les rayons restant dans un même plan. La variation de $i_{2}$ est due à l'effet d'obliquité des rayons qui présentent une différence de marche entre deux traits consécutifs multipliée par $\cos \beta / 2$ :

$$
a \cos (\beta / 2)\left(\sin i_{1}+\sin \left(i_{2}+\delta i_{2}\right)\right)=k \lambda
$$

Combinée à la même équation pour les rayons dans le plan de section principale :

$$
a\left(\sin i_{1}+\sin i_{2}\right)=k \lambda
$$

on obtient la valeur de $\delta i_{2}$ en fonction de $\beta$ supposé petit :

$$
\begin{gathered}
a \cos i_{2} \delta i_{2}=k \lambda(1 / \cos (\beta / 2)-1)=k \lambda \beta^{2} / 8 \\
\delta i_{2}=\beta^{2} / 8 k \lambda / a \cos i_{2} \\
\delta \lambda=\delta i_{2} / D_{2}=\lambda \beta^{2} / 8 .
\end{gathered}
$$

expression qui ne diffère de celle obtenue pour le prisme que par le facteur $\left(n^{2}-1\right) / n$ égal à l'unité pour n égal à 1,618 , et par le facteur dispersion du verre $\lambda \mathrm{dn} / \mathrm{d} \lambda$. 
Si l'on veut que la flèche reste inférieure à la largeur de la fente, il faut limiter la hauteur angulaire de la fente d'un spectromètre à réseau en fonction du pouvoir de résolution auquel on veut travailler :

$\beta^{2} / 8<\delta \lambda \lambda=1 / R$

$\beta<2 \sqrt{2 / R}$

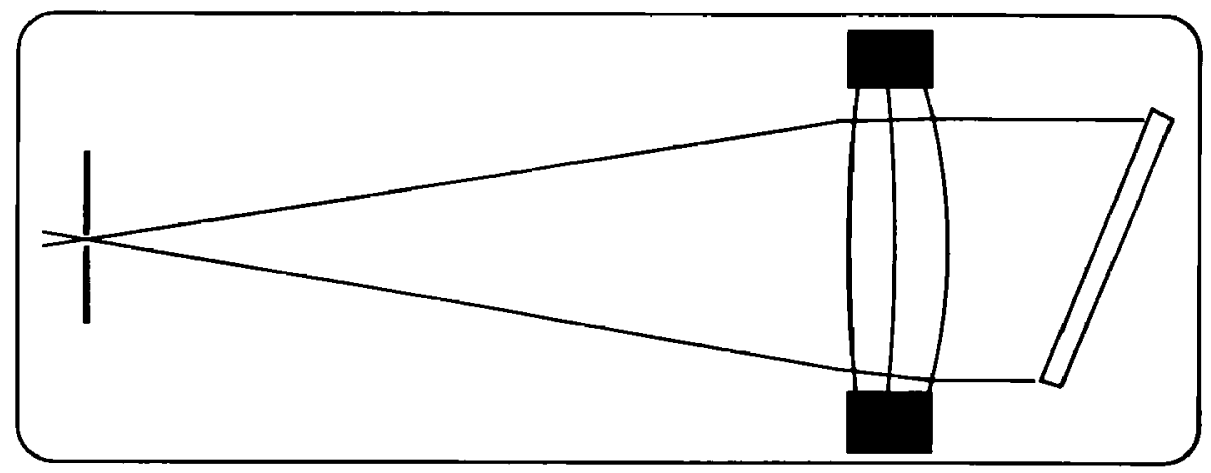

Figure 5.3 : Montage autocollimateur de Littrow

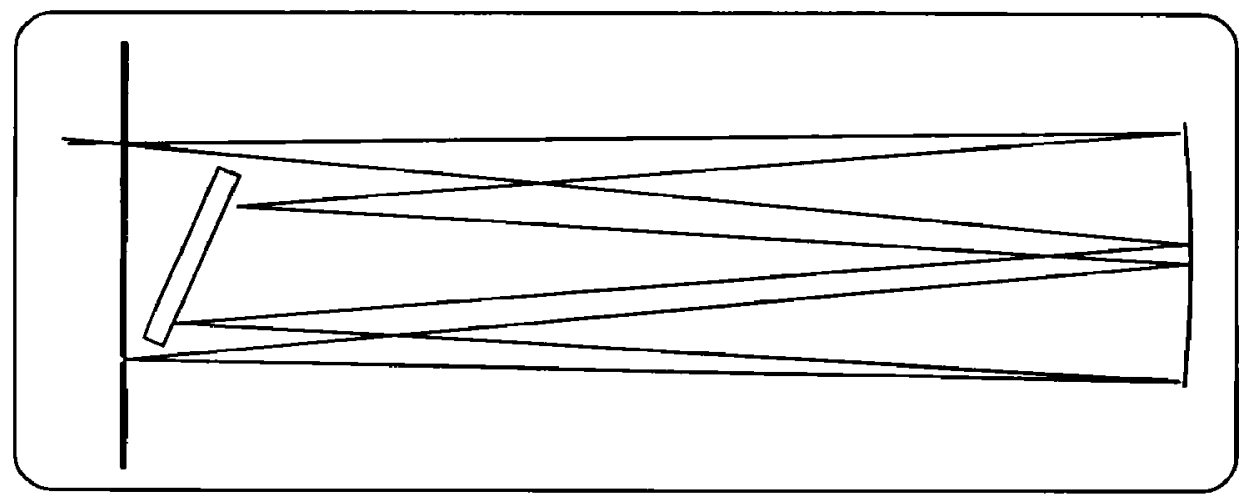

Figure 5.4 : Montage de Ebert-Fastie. 


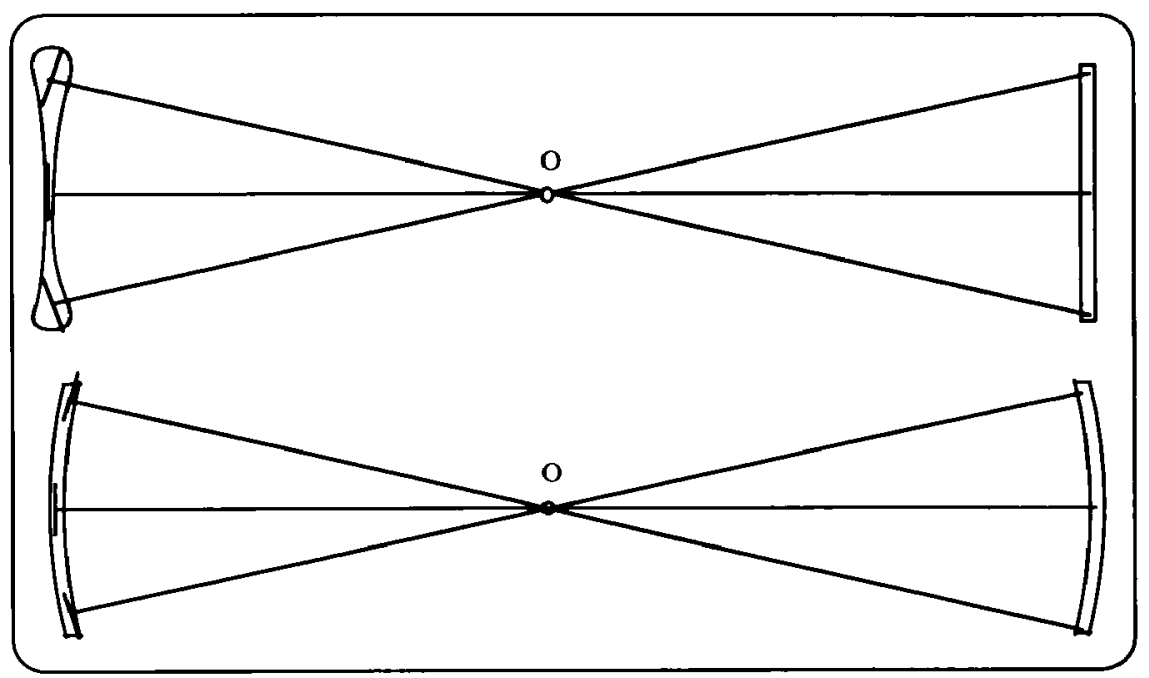

Figure 5.5 : Fentes courbes de Fastie dans un montage Ebert-Fastie. O est la trace de l'axe du miroir sphérique commun à l'entrée et à la sortie du spectromètre. En haut à gauche, image déformée par l'astigmatisme, de la fente rectiligne à droite. En bas à gauche image peu affectée par l'astigmatisme, de la fente courbe à droite.

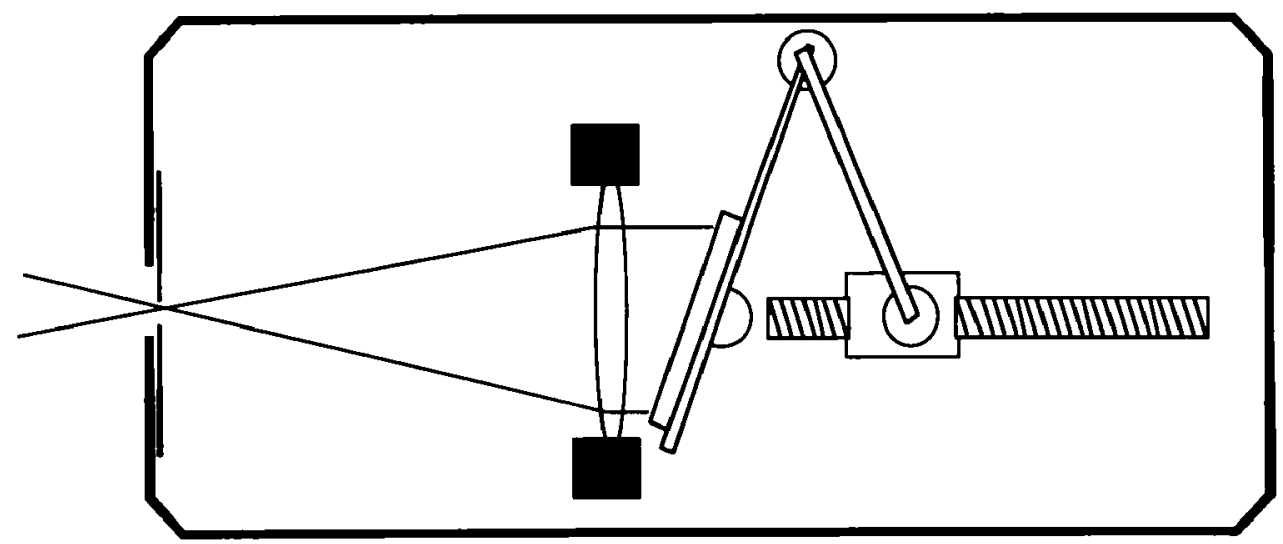

Figure 5.6 : Système de rotation du réseau à barre-sinus dans un montage auto-collimateur. La longueur d'onde affichée est proportionnelle au déplacement de l'écrou. 


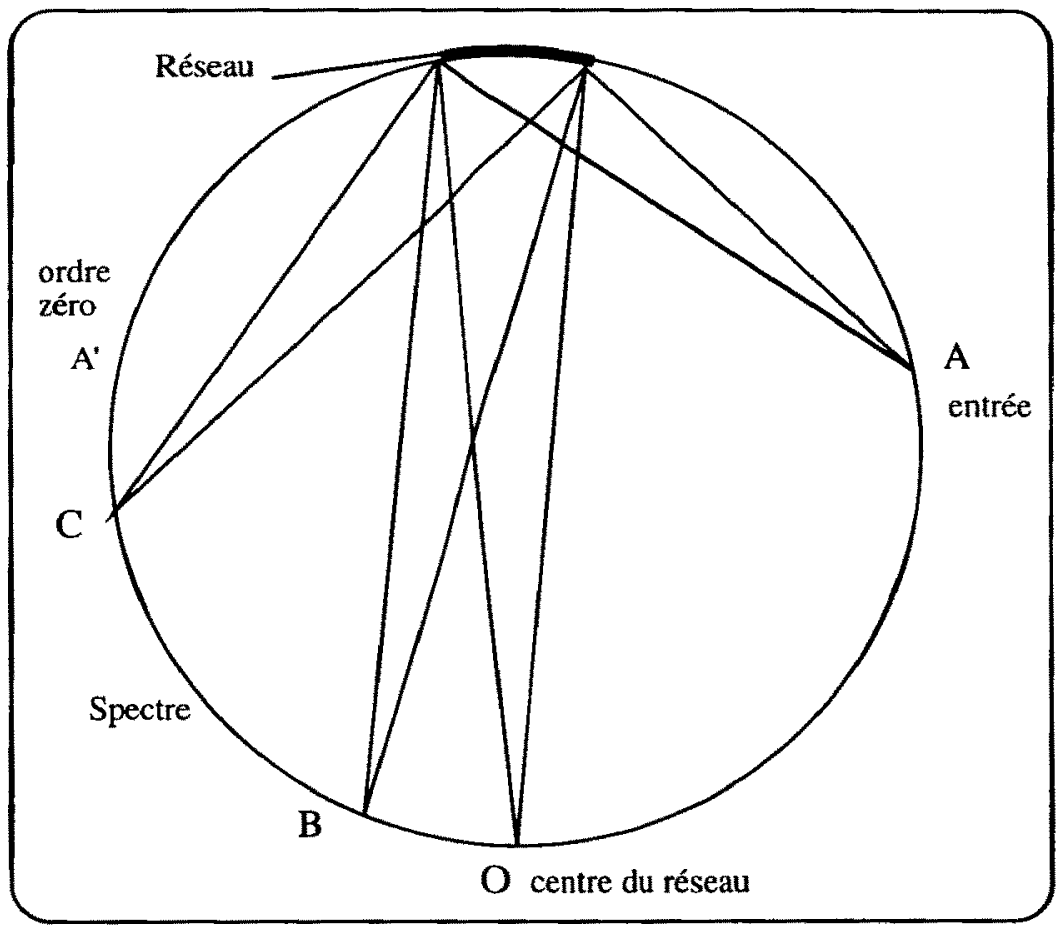

Figure 5.7 : Montage à réseau concave de Rowland.

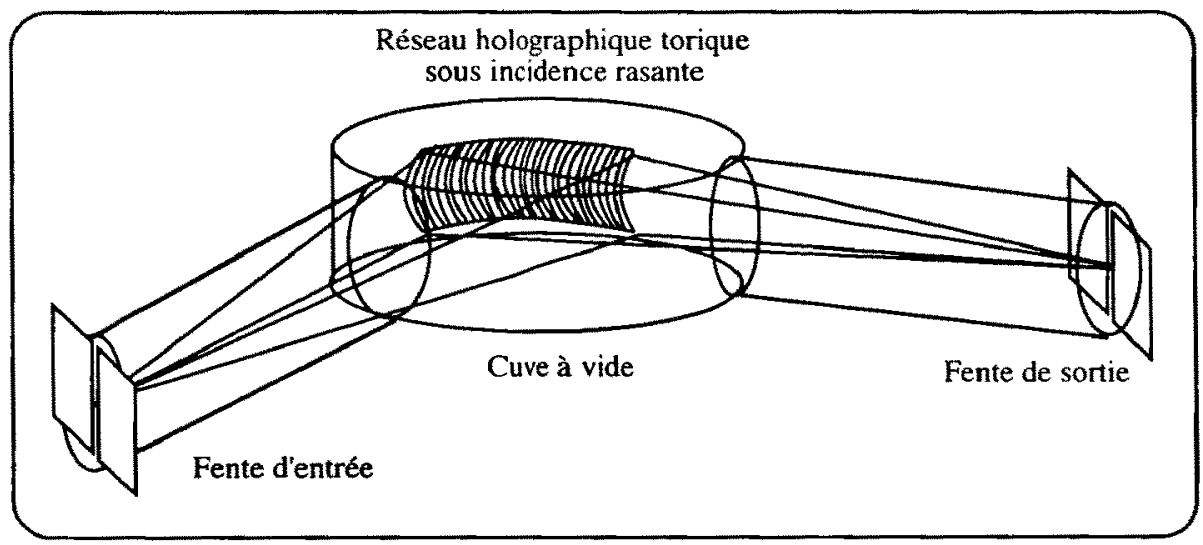

Figure 5.8 : Montage à réseau torique pour l'extrềne ultraviolet (d'uprès document Jobin \& Yvon). 


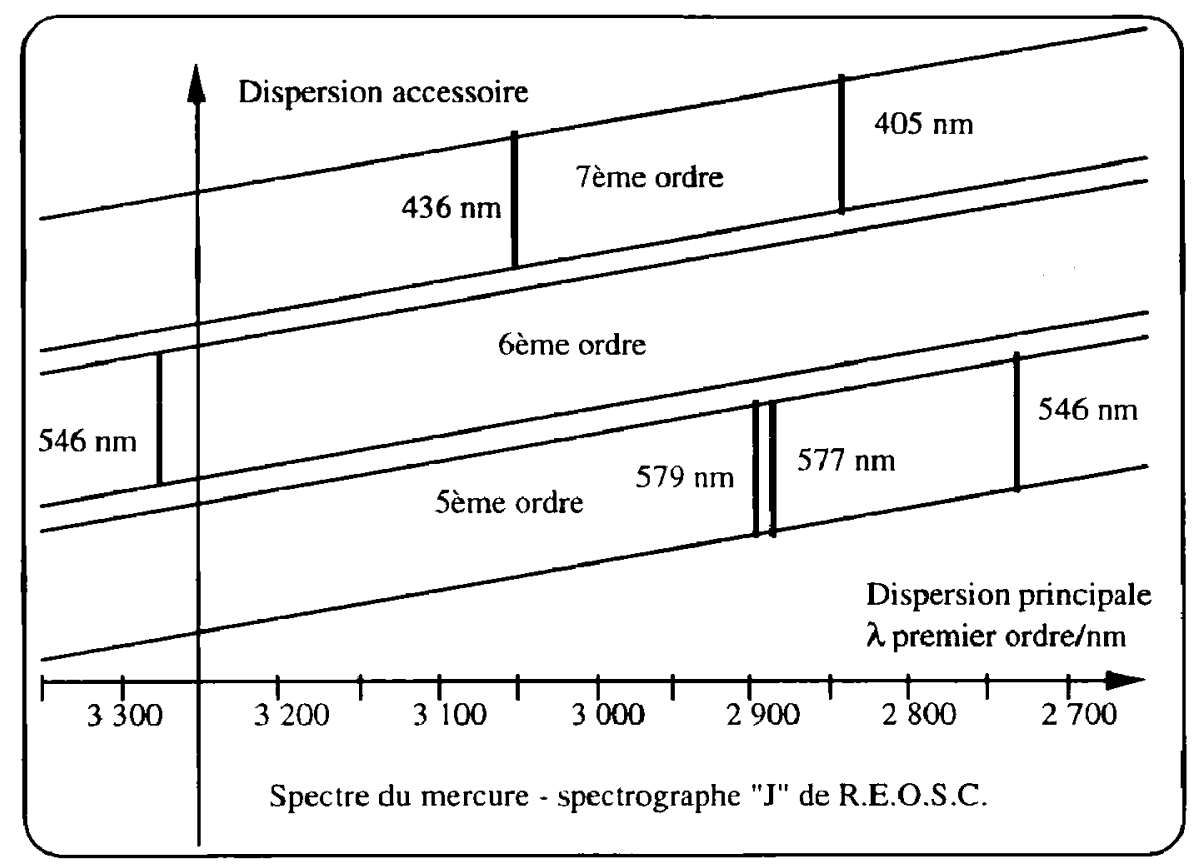

Figure 5.9 : Montage à dispersions croisées.

\section{SPECTROMÈTRE DE FABRY ET PEROT}

Finesse, intervalle spectral libre, étendue de faisceau, le Fabry-Perot sphérique. Les cavités Fabry-Perot et la mesure des fréquences optiques.

\subsection{Fonction d'appareil, limite et pouvoir de résolution}

Les interférences à ondes multiples observées entre deux miroirs plans parallèles conduisent à des résonances successives représentées par la fonction d'Airy:

$$
\frac{T}{1-R} \frac{1}{1+\frac{4 R}{(1-R)^{2}} \sin ^{2}(\varphi / 2)}
$$

où $\varphi$ représente le déphasage entre deux rayons successifs : $\varphi=2 \pi n 2 \mathrm{e} \sigma \cos \mathrm{i}$ 


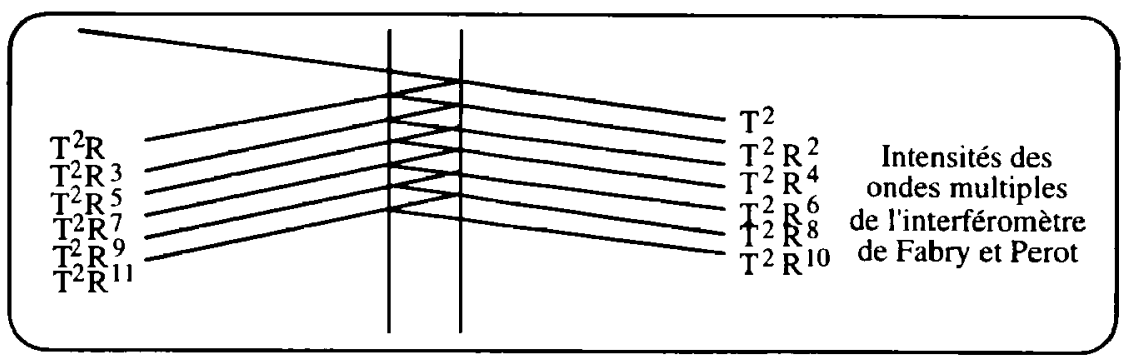

Figure 6.1 : La marche des rayons dans un interféromètre de Fabry et Perot.

Etablie en 1850 par John Biddell Airy pour interpréter le faible écart à des franges sinusoïdales observé lorsque le pouvoir réflecteur des lames est faible, c'est Charles Fabry qui a montré que cette formule prenait des allures très particulières lorsque le pouvoir réflecteur $R$ s'approche de l'unité. La finesse des franges est une des caractéristiques essentielles de ce type d'interféromètre. Elle est définie par le rapport $\Delta \sigma / \delta \sigma$ de l'intervalle entre ordres $\Delta \sigma$ à la largeur à mi-hauteur d'un ordre $\delta \sigma$. On en tire une valeur approchée de la formule d'Airy

$$
N=\frac{\pi \sqrt{R}}{1-R}
$$

et une valeur plus rigoureuse en calculant la transformée de Fourier de la réponse impulsionnelle :

$$
N=\frac{\pi}{|\log (R)|}
$$

On peut montrer que la finesse représente le nombre de réflexions efficaces par les deux miroirs. En effet $\mathrm{R}^{2 \mathrm{~N}}$ qui représente l'intensité relative du $(\mathrm{N}+1)^{\mathrm{ème}}$ rayon par rapport au premier rayon transmis est égal à $\exp (-2 \pi)=1,210^{-3}$.

Admettant $\mathrm{n}=1$ et $\mathrm{i}=0^{\circ}$, la formule d'Airy permet d'obtenir sans difficulté l'intervalle spectral libre entre deux ordres successifs $\Delta \sigma=1 / 2 \mathrm{e}$ et la largeur spectrale à mi-hauteur d'un pic de transmission $\delta \sigma=1 / 2 \mathrm{Ne}$. On en déduit le pouvoir de résolution $\mathrm{R}=\sigma / \delta \sigma=2 \mathrm{Ne} \sigma$.

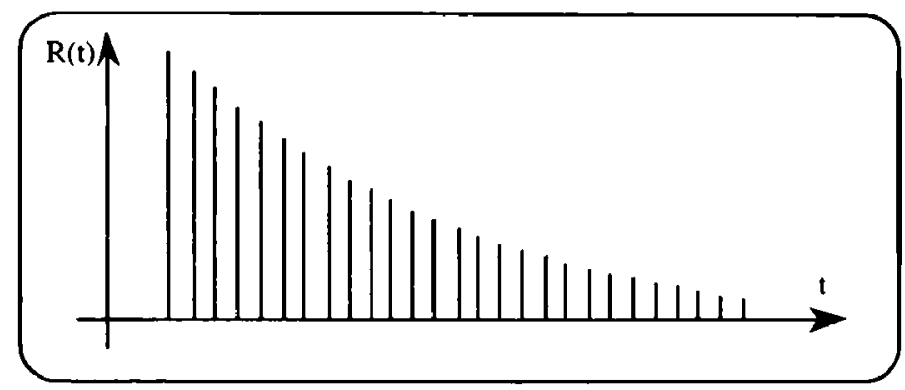

Figure 6.2 : La réponse impulsionnelle d'un spectromètre de Fabry et Perot. 


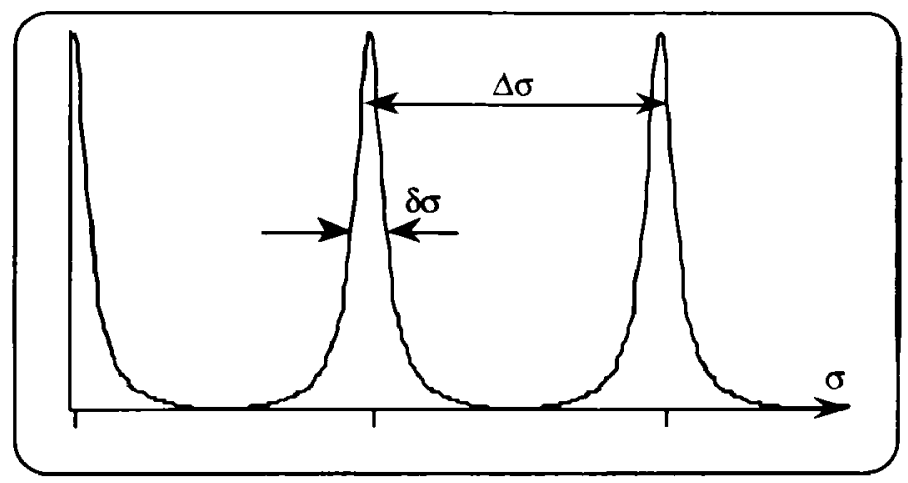

Figure 6.3 : La fonction de transfert d'un spectromètre de Fabry et Perot.

Tableau 6.1 : Les valeurs numériques de la finesse réflectrice.

\begin{tabular}{|c|c|c|c|c|c|c|c|c|}
\hline $\mathrm{R}$ & 1 & 0,99 & 0,95 & 0,90 & 0,80 & 0,70 & 0,50 & 0,30 \\
\hline$\frac{\pi \sqrt{\mathrm{R}}}{1-\mathrm{R}}$ & $\infty$ & 312,6 & 61,24 & 29,80 & 14,05 & 8,76 & 4,44 & 2,46 \\
\hline$\frac{\pi}{\log (\mathrm{R})}$ & $\infty$ & 312,6 & 61,25 & 29,82 & 14,08 & 8,81 & 4,53 & 2,61 \\
\hline
\end{tabular}

Un parallèle s'établit d'emblée avec le réseau : si l'on remarque que 2 e $\sigma$ représente l'ordre $\mathrm{k}$ de la résonance, le pouvoir de résolution d'un Fabry-Perot s'écrit $\mathrm{R}=\mathrm{Nk}$. Comme pour le réseau, $\mathrm{k}$ représente l'ordre d'interférence et $\mathrm{N}$ le nombre de rayons efficaces. Mais ici $\mathrm{N}$ est de l'ordre de quelques dizaines alors que $\mathrm{k}$ est de l'ordre de plusieurs centaines de milliers.

Le pouvoir de résolution d'un interféromètre de finesse 50 et de deux centimètres d'épaisseur est égal à $410^{6}$. Dans un encombrement très réduit, le Fabry Perot permet d'atteindre de très hauts pouvoirs de résolution. Son principal inconvénient est l'étroitesse de l'intervalle spectral libre, lié au très grand ordre d'interférence. L'association d'un interféromètre et d'un prémonochromateur à réseau permet, mais difficilement, de séparer les ordres de l'interféromètre.

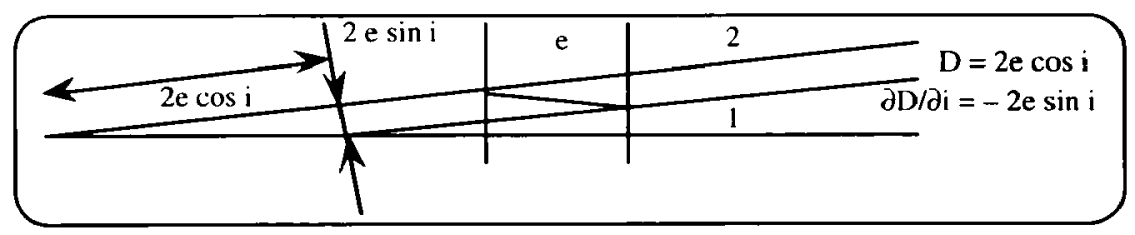

Figure 6.4 : Luminosité de l'interféromètre de Fabry et Perot. 


\section{2 Étendue de faisceau, luminosité}

Les relations utilisées pour définir la limite et le pouvoir de résolution supposent $i=0, c^{\prime}$ est-àdire un faisceau d'étendue nulle. Pour connaître l'angle solide qui peut être utilisé, il faut revenir à la structure des franges d'interférence à l'infini. La différence de marche $D=2 e \cos i$ est une fonction décroissante de $\mathrm{i}$, qui passe par un maximum pour $\mathrm{i}=0$. L'angle de phase $\varphi$ varie aussi comme cos $\mathrm{i}$. Il varie de $2 \pi$ quand on passe d'un anneau à l'anneau suivant, et de $2 \pi / \mathrm{N}$ quand on passe d'une mi-hauteur à l'autre d'un anneau. De cette constatation résulte que l'angle solide entre deux anneaux consécutifs est une constante et ne dépend que de l'épaisseur e et de la longueur d'onde de la lumière :

$$
\begin{gathered}
2 e \cos i_{k}=k \lambda \\
2 e \cos i_{k-1}=(k-1) \lambda \\
\Delta \Omega=2 \pi\left(\cos i_{k}-\cos i_{k-1}\right)=2 \pi \lambda / 2 e=2 \pi / k_{0}
\end{gathered}
$$

Le diaphragme à l'infini qui isolera un anneau brillant devra accepter une variation de $\mathrm{i}$ qui conduit à une variation de $\varphi$ égale à $2 \pi / \mathrm{N}$. Ce diaphragme pourra isoler n'importe lequel des anneaux à l'infini, puisque leurs angles solides sont tous égaux. Le plus facile à mettre en cuvre est le diaphragme qui isole l'anneau central en acceptant les angles entre $i=0$ et $i=i_{m}$ de telle sorte que l'angle solide $\Omega$ accepté corresponde à une variation totale de $\varphi$ égale à $2 \pi / \mathrm{N}$ :

$$
\Omega=2 \pi / \mathrm{Nk}_{0}=2 \pi \mathrm{R}_{0}
$$

\subsection{Comparaison avec le réseau}

Cet angle solide est de beaucoup ( 100 à 200 fois) plus grand que celui accepté par un spectromètre à réseau de même pouvoir de résolution. On peut comparer ainsi la luminosité d'un réseau et celle d'un interféromètre de Fabry et Perot :

$$
\begin{gathered}
\text { UR }_{\text {réseau }}=\lambda \beta \mathrm{HR}_{\mathrm{o}}=\beta \mathrm{HL}\left(\sin \mathrm{i}_{1}+\sin \mathrm{i}_{2}\right) \\
\mathrm{UR}_{\text {Fabry-Perot }}=2 \pi \mathrm{S} \\
\text { UR Fabry-Perot } / \mathrm{UR}_{\text {réseau }}=\frac{\beta\left(\sin \mathrm{i}_{1}+\sin \mathrm{i}_{2}\right)}{2 \pi} \approx \beta / 2 \pi .
\end{gathered}
$$

Nous avons vu que la hauteur angulaire $\beta$ d'un spectromètre à réseau ne pouvait pas excéder quelques centièmes de radian, surtout pour les pouvoirs de résolution élevés $(R>$ 100 000). Cette très grande luminosité des spectromètres de Fabry et Perot est contrariée par la faible valeur de l'intervalle spectral libre $\Delta \sigma$, ce qui oblige à associer l'interféromètre avec un prémonochromateur qui réduit considérablement l'étendue de faisceau disponible. L'usage spectrométrique du Fabry-Perot a donc été restreint au cas des très grands pouvoirs de résolution et des spectres atomiques, où le nombre de raies en émission n'est pas trop grand pour que l'on puisse isoler ces raies facilement avec un prémonochromateur. 
Les cavités Fabry-Perot ont repris le devant de l'actualité avec le développement des lasers. Ce sont des cavités de ce type qui provoquent l'oscillation dans un milieu amplificateur, et la très grande finesse de l'émission permet de travailler sur des cavités externes avec de très grands ordres d'interférence. La résonance provoque de très fortes surintensités qui permettent de renforcer les effets non linéaires tels que l'absorption saturée, les effets Raman et Brillouin stimulés. Les cavités modernes dont les miroirs sont traités avec des couches multidiélectriques de très faibles pertes (de l'ordre de quelques parties par million) peuvent offrir des finesses égales à plusieurs centaines de milliers (cavités des gyrolasers, cavités ultrastables pour l'asservissement en fréquence de lasers).

Ce sont des cavités Fabry Perot qui permettent de déceler des écarts à la résonance de quelques kilohertz avec des atomes ou des molécules sur des transitions de plusieurs centaines de térahertz. C'est grâce à de tels dispositifs que la vitesse de la lumière a pu gagner deux décimales en 1970 et devenir étalon pour la mesure des distances, et que la constante de Rydberg, qui régit toutes les transitions atomiques et moléculaires, a pu être mesurée avec une incertitude relative de quelques $10^{-11}$. Les horloges du futur s'appuieront très probablement sur des cavités optiques de type Fabry et Perot dont la résonance très aiguë sera asservie sur une transition atomique ou moléculaire.

\subsection{Fabry-Perot sphérique}

L'ordre d'interférence d'un Fabry-Perot plan est stationnaire à l'infini pour i $=0$, c'est-à-dire lorsque les rayons qui interfèrent sont superposés. La variation de la différence de marche $D$ cos $i$ avec l'angle d'incidence $i$ est proportionnelle à $\mathrm{D} \sin \mathrm{i}$, qui est la distance entre les rayons parallèles qui interfèrent à l'infini (Fig. 6.4). C'est pourquoi les anneaux sont de plus en plus fins et serrés quand leur rayon angulaire augmente.

$\mathrm{P}$. Connes a proposé le Fabry-Perot sphérique dont les deux miroirs sphériques confocaux ont chacun leur centre de courbure sur le sommet de l'autre. On peut vérifier que tout rayon incident donne dans l'approximation de Gauss des rayons émergents superposés (Fig. 6.5). La différence de marche est ainsi stationnaire pour toute valeur de $i$, donnant une teinte plate à l'infini, et un angle solide $\Omega$ très supérieur à celui du Fabry-Perot plan.

De plus une petite rotation d'un des miroirs déplace son centre sur l'autre miroir sans dérégler l'interféromètre, qui est beaucoup plus stable qu'un Fabry-Perot plan. Ce dispositif n'est toutefois avantageux que pour des épaisseurs suffisantes (plusieurs centimètres).

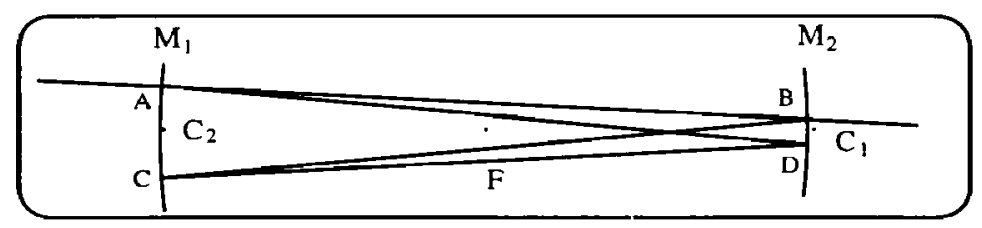

Figure 6.5 : Cavité Fabry-Perot sphérique à champ compensé : $D=4 \mathrm{e}=4$ fois le rayon des miroirs $M_{1}$ et $M_{2}$, $\partial \mathrm{D} / \partial \mathrm{i}=0$. La cavité est stable.

\section{SPECTROMÉTRIE PAR TRANSFORMATION DE FOURIER}

Principe, limite et pouvoir de résolution, apodisation, angle solide, échantillonnage de l'interférogramme, avantage multiplexe. 


\subsection{Petite histoire de la spectrométrie de Fourier}

La première tentative de spectrométrie par transformation de Fourier remonte à Albert Michelson qui, lorsqu'il est venu à Sèvres au Bureau International des Poids et Mesures pour faire la mesure du mètre en longueurs d'onde, rechercha les radiations atomiques les plus monochromatiques. Observant visuellement les variations du facteur de visibilité données par une lampe à mercure en fonction de la différence de marche il fit la première observation d'une structure isotopique. Comme il n'enregistrait pas les franges il en perdait la phase, ce qui lui laissa une ambiguité sur l'asymétrie du profil, mais cette asymétrie mise à part le profil spectral qu'il dessina dans son mémoire est tout à fait ressemblant.

Ce furent ensuite $P$. Jacquinot et $P$. Fellgett qui proposèrent ensemble et indépendamment d'analyser par transformation de Fourier l'interférogramme à deux ondes donné par une source pour retrouver la distribution spectrale de sa puissance. C'était alors le tout début des calculateurs numériques dont les performances étaient encore très limitées tant en capacité de mémoire qu'en vitesse de calcul.

J.Connes fit dans le cadre de sa thèse une étude théorique approfondie de la méthode illustrée par un spectre spectaculaire : le spectre du ciel nocturne dans le proche infrarouge. Ses travaux furent repris par son époux Pierre Connes qui, avec de nombreux collaborateurs comme Jacques Pinard, Guy Guelachvili, Guy Michel et quelques autres développèrent les interféromètres et les systèmes d'asservissement associés pendant que Janine Connes et Hervé Delouis mettaient à profit les progrès dans le domaine des calculateurs et des algorithmes de calcul. Il fallait plusieurs heures à l'ordinateur IBM de la Place Vendôme pour que Janine Connes calcule la transformée de Fourier d'un interférogramme de 10000 points, et la probabilité de panne d'une machine à lampes sur cette durée était très élevée. Il faut moins d'une minute à une machine courante pour calculer le spectre d'un million de points, et c'est le tracé du spectre comportant un très grand nombre d'élément spectraux qui est aujourd'hui de beaucoup l'opération la plus longue.

A ses débuts la spectrométrie de Fourier est apparue comme une méthode indirecte, par laquelle on enregistrait d'abord un interférogramme qui était ensuite transformé a posteriori sur un gros ordinateur, par opposition aux spectromètres à prisme ou à réseau qui permettaient d'enregistrer directement le spectre. La transformation de Fourier est en réalité l'opération mathématique qui effectue l'analyse spectrale d'un signal quelconque. L'interférométrie n'est qu'un artifice pour transposer dans le domaine des très basses fréquences (quelques hertz) les très hautes fréquences optiques (plusieurs centaines de térahertz).

\subsection{Principe}

La spectrométrie par transformation de Fourier commence par l'enregistrement du signal d'interférence à deux ondes donné par la source dans un interféromètre de Michelson :

$$
F(D)=\int F_{\sigma}(1+\cos 2 \pi \sigma D) d \sigma
$$

dont la partie variable I(D) avec la différence de marche D est la transformée de Fourier en cosinus de la densité spectrale de puissance $F_{\sigma}$. On revient donc à cette densité spectrale, le spectre de la source, par la transformation de Fourier inverse :

$$
F_{\sigma}=\int I(D) \cos (2 \pi \sigma D) d D
$$

Pour établir la forme de la fonction d'appareil, nous éclairons l'interféromètre avec une radiation monochromatique de nombre d'ondes $\sigma$. L'interférogramme est une sinusoìde

$$
I(D)=\left(F_{0} / 2\right) \cos \left(2 \pi \sigma_{o} D\right)
$$


Le spectre calculé s'écrit :

$$
\begin{gathered}
S(\sigma)=\int_{0}^{D_{F}} I(D) \cos (2 \pi \sigma D) d D= \\
\left(F_{o} D_{m} / 2\right)\left(\frac{\sin \left(2 \pi\left(\sigma-\sigma_{0}\right) D_{m}\right)}{2 \pi\left(\sigma-\sigma_{0}\right) D_{m}}+\frac{\sin \left(2 \pi\left(\sigma+\sigma_{0}\right) D_{m}\right)}{2 \pi\left(\sigma+\sigma_{0}\right) D_{m}}\right)
\end{gathered}
$$

C'est une fonction paire qui comporte un pic en forme de sinus cardinal centré en $\sigma=\sigma_{\mathrm{o}}$ et un pic centré en $\sigma=-\sigma_{0}$. Le spectre des nombres d'ondes négatifs ne correspond bien sûr à aucune réalité physique (il est dû au fait que la transformation de Fourier en cosinus ne donne que la partie paire du spectre) et sera tout d'abord ignoré. Il faudra y penser avec les problèmes d'échantillonnage dont une conséquence est le repliement des spectres.

La largeur à mi-hauteur de la fonction d'appareil est à peu près égale à la largeur entre le maximum et le premier zéro, soit $\delta \sigma \approx 1 / 2 \mathrm{D}_{\mathrm{m}}$. Mais la forme de la fonction d'appareil obtenue par calcul de la transformée de Fourier de l'interférogramme est très contestable : elle comporte des valeurs négatives incompatibles avec une densité spectrale de puissance et des pieds gênants dans l'observation de raies faibles au voisinage de raies intenses.

\subsection{Apodisation de la fonction d'appareil}

L'amplitude de ces pieds est liée à la discontinuité de l'interférogramme qui est brutalement interrompu à la différence de marche $\mathrm{D}_{\mathrm{m}}$. C'est le phénomène de Gibbs, bien connu des praticiens du signal dans les systèmes linéaires. Pour atténuer les pieds de la fonction d'appareil, une technique efficace consiste à multiplier l'interférogramme par une fonction décroissante de la différence de marche de telle sorte que l'amplitude de l'interférogramme s'annule sans discontinuité à $\mathrm{D}=\mathrm{D}_{\mathrm{m}}$. La plus simple des méthodes consiste à multiplier l'interférogramme par la fonction $F(D)=1-D / D_{m}$ pour $0<D<D_{m}$. L'interférogramme est alors multiplié par une fonction triangle, produit d'autocorrélation de deux fonctions rectangles de largeur $\mathrm{D}_{\mathrm{m}} / 2$. La transformée de Fourier de cet interférogramme atténué est le pioduit de convolution des deux pics de Dirac centrés en $\sigma_{\mathrm{o}}$ et en $-\sigma_{0}$ par le carré de la transformée de Fourier d'une fonction rectangle de largeur $D_{m} / 2$. C'est un sinus cardinal au carré, dont les zéros sont deux fois plus éloignés du pic que la fonction précédente. On admet que la largeur à mi-hauteur de cette nouvelle fonction d'appareil apodisée est $\delta \sigma=1 / \mathrm{D}_{\mathrm{m}}$, ce qui conduit à l'expression du pouvoir de résolution de la spectrométrie par transformation de Fourier :

$$
R_{\mathrm{o}}=\sigma / \delta \sigma=\sigma \mathrm{D}_{\mathrm{m}}=\mathrm{D}_{\mathrm{m}} \lambda
$$

\subsection{Angle solide disponible, luminosité}

Les calculs qui précèdent sont faits en supposant des rayons parallèles, donc un faisceau d'étendue nulle. Si nous admettons dans l'interféromètre un faisceau qui aura la symétrie de révolution des anneaux à l'infini autour de la normale aux miroirs, en désignant par $i_{m}$ l'angle maximum admis dans l'interféromètre, le flux à la sortie de l'interféromètre s'écrit pour une source monochromatique de nombre d'ondes $\sigma_{0}$ : 


$$
\begin{gathered}
F(D)=\frac{L_{0}}{2} \int_{0}^{\Omega}\left(1+\cos \left(2 \pi \sigma_{0} D \cos i\right)\right) d \Omega \quad \text { avec } \Omega=2 \pi\left(1-\cos i_{m}\right) \\
F(D)=\frac{L_{0}}{2} \int_{\operatorname{cosi}}^{1}\left(1+\cos \left(2 \pi \sigma_{0} D \cos i\right)\right) 2 \pi d \cos i=L_{0} \frac{\Omega}{2}\left(1+\frac{\sin \left(\sigma_{0} \Omega D / 2\right)}{\sigma_{0} \Omega D / 2} \cos \left(2 \pi \sigma_{0} D\left(1-\frac{\Omega}{4 \pi}\right)\right) .\right.
\end{gathered}
$$

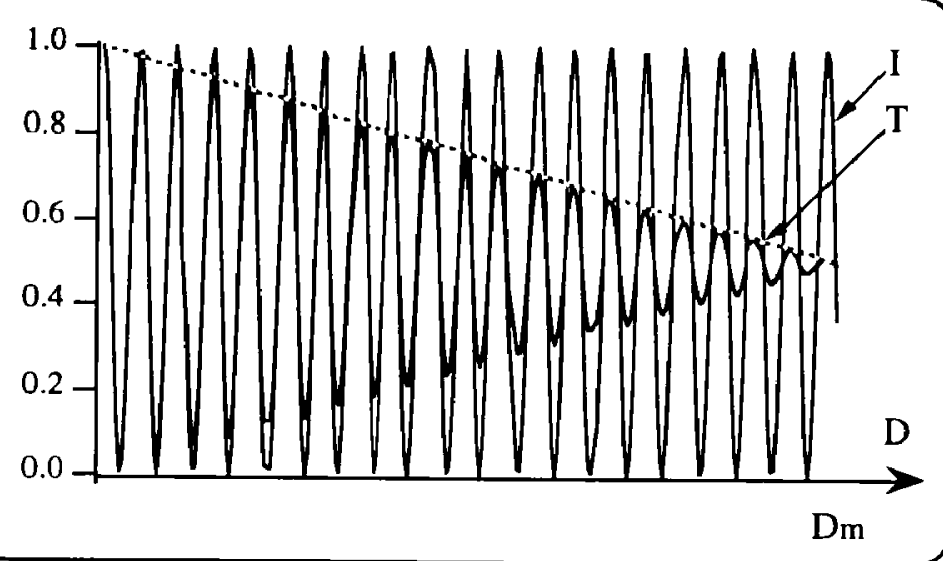

Figure 7.1 : Interférogrammes brut (I) et atténué (T) pour éviter le phénomène de Gibbs et apodiser la fonction d'appareil.

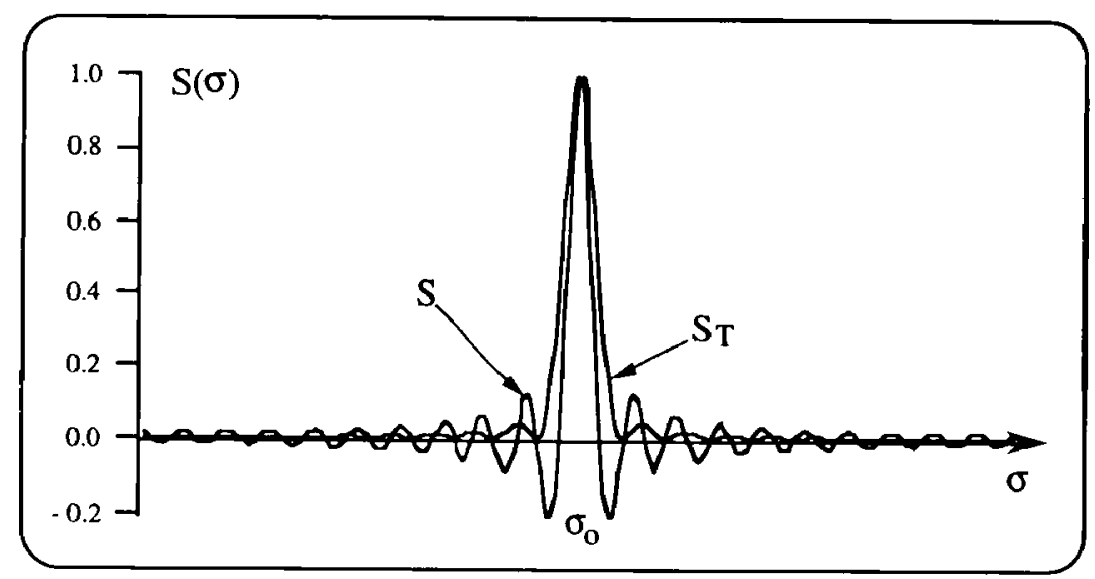

Figure 7.2 : Fonctions d'appareil brute (S) et apodisée $\left(\mathrm{S}_{\mathrm{T}}\right)$ correspondant aux interférogrammes de la figure 7.1 . 


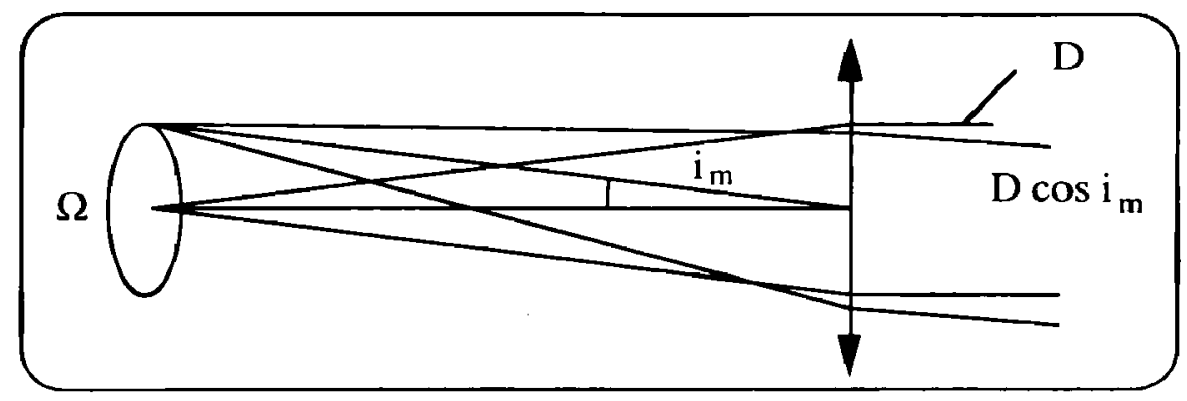

Figure 7.3 : Angle solide $\Omega$ du diaphragme à l'infini qui éclaire l'interféromètre.

L'angle solide $\Omega$ du faisceau se traduit par deux effets de natures très différentes :

$\left.1^{\circ}\right)$ La différence de marche apparente est multipliée par un facteur d'obliquité $1-\Omega / 4 \pi=$ $\left(1+\cos i_{m}\right) / 2$ qui est la moyenne entre la valeur au centre $D$ et la valeur au bord du champ $D$ $\cos i_{m}$.

$2^{\circ}$ ) L'amplitude de l'interférogramme est multipliée par un facteur de visibilité égal à 1 pour $\mathrm{D}=0$ et décroissant lentement avec $\mathrm{D}$. Ce facteur s'annule pour

$$
\mathrm{D}_{\mathrm{m}}=2 \pi / \sigma_{\mathrm{o}} \Omega
$$

Au-delà de cette différence de marche le facteur de visibilité est négatif, ce qui se traduit par le fait que la sinusoïde est en opposition de phase avec le début de l'interférogramme. Dans le calcul ultérieur de la transformée de Fourier de l'interférogramme, la partie des franges située au-delà de $D_{m}$ donnera un terme négatif à l'amplitude de la composante $\sigma_{0}$. Il est intuitif que l'on ne gagne rien à prolonger l'enregistrement de l'interférogramme au-delà de $\mathrm{D}_{\mathrm{m}}$.

Il en résulte une relation simple entre l'angle solide $\Omega$ et le pouvoir de résolution $R=$ $\sigma_{\mathrm{o}} \mathrm{D}_{\mathrm{m}}$ recherché :

$$
\Omega \mathrm{R}=2 \pi
$$

Nous retrouvons une relation analogue à celle de l'interféromètre de Fabry et Perot. Par ailleurs la décroissance de l'interférogramme ainsi introduite par la fonction de visibilité est un excellent facteur d'apodisation, la fonction d'appareil devenant le produit de convolution d'une fonction sinus cardinal de largeur $\delta \sigma_{1}=1 / 2 D_{m}$ par une fonction rectangle de largeur $\delta \sigma_{2}=1 / \mathrm{D}$.

Note : certains expérimentateurs ont enregistré des interférogrammes très au-delà de $D_{m}$ dans un but bien précis. On se trouve avec une fonction d'apodisation qui est la transformée de Fourier d'un profil rectangulaire, et la fonction d'appareil se rapproche d'une fonction porte aux flancs abrupts et dénuée de pieds. Mais la limite de résolution n'est plus déterminée par la différence de marche maximale atteinte : elle ne dépend plus que de l'angle solide $\Omega$ du diaphragme à l'infini qui éclaire l'interféromètre. 


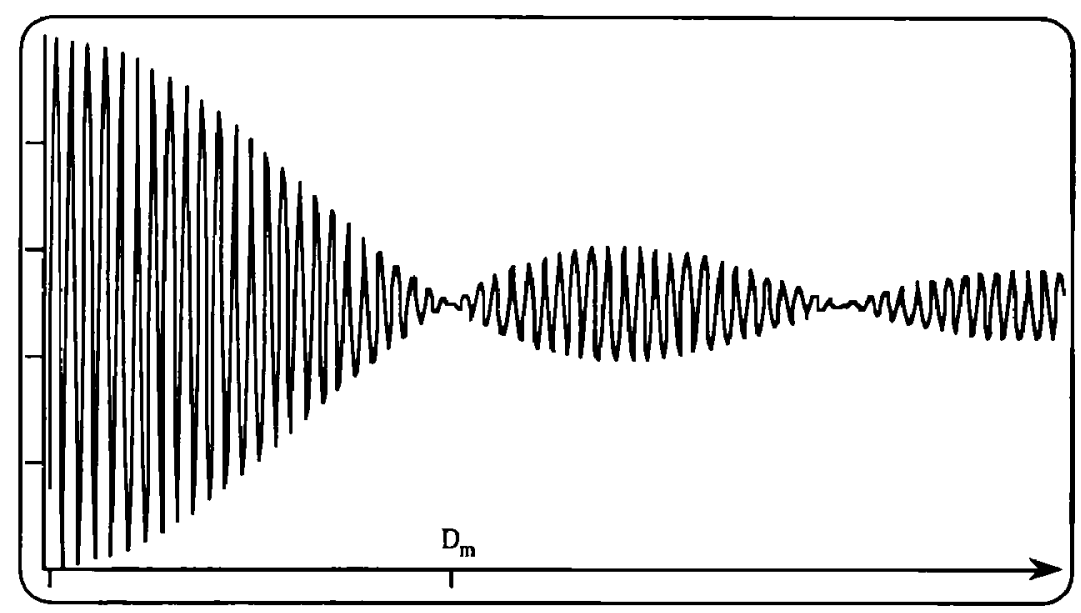

Figure 7.4 : Interférogramme enregistré avec un angle solide $\Omega$. Au-delà de la différence de marche $D_{m}$, l'interférogramme est en opposition de phase (facteur de visibilité négatif dans le premier lobe de la fonction sinc).

\section{5 Échantillonnage de l'interférogramme}

Pour calculer la transformée de Fourier de l'interférogramme, quelques tentatives ont été faites par des méthodes analogiques (filtres électroniques, diffraction en lumière cohérente) mais seule le calcul numérique a permis d'atteindre de hauts pouvoirs de résolution. Il faut donc échantillonner et numériser l'interférogramme. De même que l'échantillonnage de la pupille d'un réseau par ses traits nous a conduits à la multiplicité des ordres, de même l'échantillonnage de l'interférogramme fait apparaître des ordres parasites dans le spectre. Mais ici le problème se complique à cause de l'existence de fréquences négatives dans la fonction d'appareil, dont nous n'avons plus parlé depuis qu'elle est apparue.

Multiplier l'interférogramme par un peigne de Dirac de pas p revient à convoluer le spectre par un peigne de Dirac de pas 1/p. Mais l'existence du spectre image conduit à un recouvrement du spectre des fréquences négatives sur le spectre des fréquences positives, appelé repliement de spectre, qui complique le problème du recouvrement des ordres. Un spectre qui s'étend entre $\sigma_{\min }$ et $\sigma_{\max }$ occupe un intervalle spectral $-\sigma_{\max }, \sigma_{\max }$ égal à 2 $\sigma_{\max }$. La condition de non recouvrement des spectres s'écrit donc :

$$
1 / \mathrm{p}>2 \sigma_{\max } \quad \mathrm{p}<\lambda_{\mathrm{m}} / 2
$$

où $\lambda_{m}$ est la longueur d'onde minimale présente dans le spectre. Nous retrouvons ici le bien connu théorème de Shannon.

Lorsque l'intervalle spectral $\sigma_{\max }-\sigma_{\min }$ est étroit, on occupe très mal l'intervalle spectral disponible. J. Connes a montré que l'on pouvait alors obéir à une forme moins sévère du théorème de Shannon qu'elle a appelé le théorème de Shannon généralisé : le repliement de spectre ne se répercute pas sur le domaine spectral $\sigma_{\max }-\sigma_{\min }$ si les limites sont convenablement situées et en choisissant $\mathrm{p}<\frac{2}{\sigma_{\max }-\sigma_{\min }}$. 


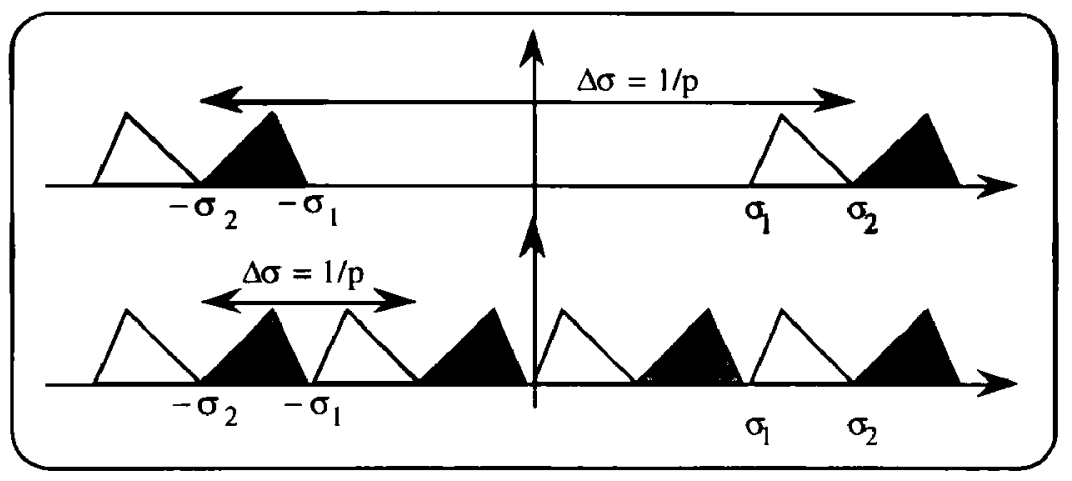

Figure 7.6: Le théorème de Shannon et sa version généralisée appliqués à un spectre qui s'étend entre $\sigma_{1}=$ $2000 \mathrm{~cm}^{-1}$ et $\sigma_{2}=3000 \mathrm{~cm}^{-1}$, avec $p=1,67 \mu \mathrm{m}$ et $p=5 \mu \mathrm{m}$ respectivement.

\subsection{Contrôle de la différence de marche}

Une des difficultés essentielles de la spectrométrie par transformation de Fourier est de mesurer la différence de marche $\mathrm{D}$ avec une incertitude très inférieure à la longueur d'onde, pour enregistrer correctement la phase de l'interférogramme. Ce résultat n'a pu être obtenu que par l'enregistrement simultané dans le même interféromètre d'un interférogramme de référence donné par un laser stabilisé en fréquence. Du coup la difficulté s'est transformée en un avantage métrologique fondamental : la transformation de Fourier effectuée ultérieurement sur l'interférogramme en s'appuyant sur les franges du laser de référence est une comparaison directe arithmétique entre tous les nombres d'ondes $\sigma$ du spectre calculé et le nombre d'ondes $\sigma_{0}$ du laser de référence. L'exactitude des nombres d'ondes calculés par transformation de Fourier est ainsi de beaucoup supérieure à celle de toutes les méthodes spectrométriques connues.

C'est Guy Guélachvili qui en fit une très belle démonstration en 1970 dans sa thèse sur le spectre des oxydes de carbone, retrouvant en termes de nombres d'ondes des paramètres de cette molécule très bien connus en termes de fréquences par résonance paramagnétique électronique. Ce résultat le conduisit à une valeur de la vitesse de la lumière dans le vide qui aurait été la meilleure sans la mesure faite au même moment aux États-Unis grâce au laser hélium néon stabilisé en fréquence par absorption saturée dans le méthane.

\subsection{Gain en rapport signal sur bruit lié au caractère multiplexe de la méthode}

Une autre caractère essentiel de la spectrométrie par transformation de Fourier est son principe multiplexe, sur lequel avait insisté P.B. Fellgett : tous les éléments spectraux sont enregistrés simultanément pendant tout le temps de la mesure, conduisant à une réduction des bruits blancs du détecteur (bruits thermiques, bruit des rayonnements parasites) par un facteur de l'ordre de la racine carrée du nombre d'éléments spectraux.

Cet avantage fut essentiel dans les premiers résultats obtenus en astronomie par $P$. Connes et ses collaborateurs sur les spectres du proche infrarouge des planètes Mars, Jupiter, Saturne, Mercure et Vénus. Un gain 100 fut obtenu sur le pouvoir de résolution, ce qui aurait été possible avec les spectromètres à réseaux. Mais gagner 100 sur le pouvoir de résolution 
avec un spectromètre à fentes fait perdre 10000 sur le flux transmis lorsque la source présente un spectre continu. Pour garder le même rapport signal sur bruit, le bruit venant essentiellement du détecteur, il aurait fallu multiplier par $10^{8}$ la durée consacrée à un seul élément spectral. Comme il y a 100 fois plus d'élément spectraux, il fallait donc multiplier le temps total de mesure par $100 \times 10^{4} \times 10^{4}=10^{10}$.

Le développement des détecteurs infrarouge multicanaux a diminué l'impact de cet avantage, mais la spectrométrie par transformation de Fourier reste l'une des techniques les plus puissantes pour atteindre de hauts pouvoirs de résolution avec des sources peu intenses. C'est la spectrométrie qui permet d'atteindre des précisions inégalées dans la détermination des constantes physiques moléculaires.

\section{LE POUVOIR DE RÉSOLUTION THÉORIQUE DES SPECTROMÈTRES OPTIQUES.}

\subsection{Principe d'incertitude temps-fréquence.}

La fréquence d'un signal parfaitement sinusoïdal mais limité à un intervalle de temps de durée finie $T$ est l'objet d'une indétermination très fondamentale exprimée par le principe d'incertitude de Heisenberg. Nous pouvons dire que cette indétermination est mathématique, elle vient de la relation qui existe entre la largeur temporelle d'un signal et la largeur spectrale de sa transformée de Fourier. Le produit de la durée $T$ par la largeur spectrale $\delta$ est limité inférieurement par la relation :

$$
\text { T. } \delta f>1
$$

Il en résulte que l'incertitude relative off/f est au moins égale à

$$
1 /(\mathbf{f} . \mathrm{T})=1 / \mathrm{N}
$$

où $\mathrm{N}$ est le nombre de périodes du signal de fréquence $f$ observées pendant la durée $\mathrm{T}$. Cette incertitude est tout simplement l'incertitude de quantification sur le comptage des périodes du signal pendant la durée $T$. Les théories quantiques du signal conduisent à diviser cette quantité par la racine carrée du nombre de photons détectés, et tiennent ainsi compte du rapport signal sur bruit. Nous laisserons cet aspect de côté, nous refusant à interpoler à l'intérieur de la fonction d'appareil, ou à faire de la superrésolution par des traitements de signaux adaptés.

\subsection{Transposition au domaine optique.}

Dans un spectromètre optique, la mesure directe des fréquences est impossible parce que les détecteurs ne sont pas assez rapides pour suivre les variations temporelles de l'amplitude lumineuse et compter des périodes de l'ordre de 2 femtosecondes, soit $2.10^{-15} \mathrm{~s}$. La distinction entre deux fréquences optiques différentes se fera en étudiant les variations de l'amplitude résultant de la superposition de deux ou plusieurs ondes déphasées les unes par rapport aux autres par un retard $\delta$ t. Ce retard est provoqué par un chemin optique $D$ supplémentaire suivi par au moins une onde. Le nombre de périodes écoulées pendant ce retard va jouer pour la spectrométrie optique le rôle de la limitation temporelle de l'observation de la phase d'un signal sinusoïdal, et l'incertitude relative sur la détermination de la fréquence optique sera limitée inférieurement par l'inverse du nombre de périodes comptées sur $\delta t$.

Ce nombre est aussi le nombre de longueurs d'onde contenues dans le chemin optique supplémentaire D : 


$$
N=f . \delta t=D / \lambda
$$

L'incertitude relative sur la fréquence est donc traduite par l'incertitude sur le nombre $\mathbf{N}$ de longueurs d'onde observées, qui est une incertitude de comptage égale à \pm 1 , soit $1 / \mathrm{N}$ en valeur relative.

En réalité la plus petite variation de longueur d'onde que le dispositif pourra déceler est la variation qui induira une variation de une unité sur $\mathrm{N}$, l'ordre d'interférence. Cette variation provient bien sûr de la variation de $\lambda$, mais elle peut aussi être due à une variation chromatique de $\mathrm{D}$. La condition de résolution de deux longueurs d'onde distinctes de $\delta \lambda$ deviendra donc :

$$
\mathrm{dN}= \pm 1=\delta \lambda \partial / \partial \lambda(\mathrm{D} / \lambda)=-\left(\mathrm{D} / \lambda^{2}\right) \delta \lambda+(1 / \lambda) \partial \mathrm{D} / \partial \lambda
$$

De cette équation on tire :

$$
\mathrm{R}_{\mathrm{o}}=\lambda \delta \lambda=\mathrm{ID} / \lambda-\partial \mathrm{D} / \partial \lambda
$$

L'expression ainsi obtenue pour le pouvoir de résolution théorique est plus compliquée que le principe d'incertitude temps fréquence. Mais elle montre la possibilité de résoudre deux fréquences différentes même dans un dispositif n'introduisant aucun retard entre les amplitudes qui interfèrent, c'est-à-dire un dispositif travaillant dans l'ordre zéro. Il suffit pour que ce dispositif distingue deux fréquences, que ce retard nul soit chromatique. La variation de chemin optique liée à une variation de fréquence de l'onde électromagnétique induira une variation de l'état interférentiel, même à différence de marche nulle. C'est ainsi que la dispersion du verre d'un prisme permet de séparer spatialement deux fréquences différentes, bien que le prisme forme une image de fente dans l'ordre zéro.

\subsection{Applications aux différents spectromètres.}

Dans un spectromètre à prisme la différence de marche entre les rayons extrêmes qui traversent le prisme au voisinage de l'arête et au voisinage de la base est nulle. Ce résultat est une conséquence directe des relations de Descartes (figure 8.1).

$$
\mathrm{D}=0
$$

Mais le trajet suivi par le rayon qui passe près de l'arête est indépendant de la longueur d'onde, alors que le trajet du rayon qui passe à la base du prisme d'épaisseur e est n.e; ce trajet dépend de la longueur d'onde par l'indice. On vérifie bien :

$$
R_{o}=\partial \mathrm{D} / \partial \lambda=\mathrm{e} . \partial \mathrm{n} / \partial \lambda
$$

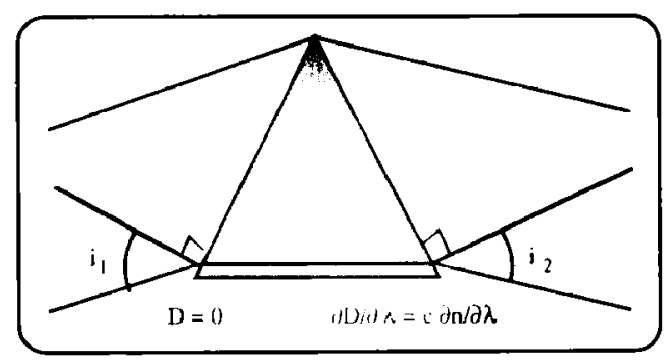

Figure 8.1 : Pouvoir de résolution théorique du prisme 
Dans un spectromètre à réseau, la différence de marche maximale entre le rayon diffracté par le premier trait et celui diffracté par le demier trait est

$$
D=L\left(\sin i_{1}+\sin i_{2}\right)
$$

$$
\text { d'où } \quad R_{0}=D / \lambda=L\left(\sin i_{1}+\sin i_{2}\right) / \lambda=N . k
$$

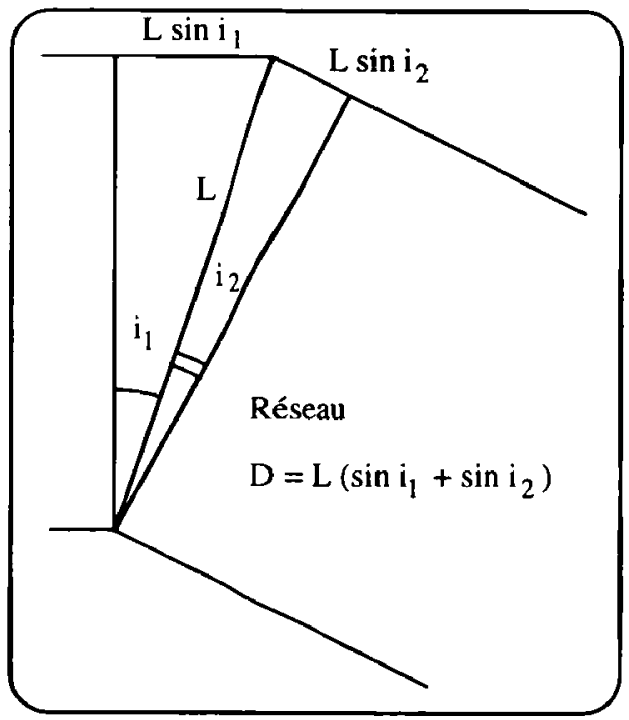

Figure 8.2 : Pouvoir de résolution théorique du réseau.

Dans un spectromètre de Fabry et Perot on peut admettre que la différence de marche maximale efficace est la différence de marche entre le premier et le $(\mathrm{N}+1)$ ième rayon transmis, ou $\mathrm{N}$ est la finesse réflectrice. Le pouvoir de résolution théorique est donc :

$$
\mathrm{R}_{\mathrm{o}}=\mathrm{D}_{\mathrm{m}} / \lambda=2 \mathrm{~N} \cdot \mathrm{e} / \lambda
$$

En spectrométrie par transformation de Fourier le retard maximum disponible est celui donné par l'interféromètre à la différence de marche maximale $\mathrm{D}_{\mathbf{m}}$.

$$
\mathrm{R}_{\mathrm{o}}=\mathrm{D}_{\mathrm{m}} \lambda
$$

Il est un dispositif où apparaissent simultanément les deux termes de l'expression générale de $R_{o}$ donnée ci-dessus : c'est l'échelon de Michelson, un réseau à petit nombre de traits qui combine les interférences entre ondes issues de marches successives et la dispersion apportée par les lames de verre constituant ces marches. Si e est l'épaisseur d'une marche, n l'indice du verre constituant les lames, et $\mathrm{N}$ le nombre de lames, l'étude de la dispersion angulaire de l'échelon et de la largeur de sa tache de diffraction conduit à l'expression du pouvoir de résolution théorique :

$$
R_{0}=\mathrm{Ne}(\mathrm{n}-1) / \lambda-\mathrm{Ne} \partial \mathrm{n} / \partial \lambda
$$


On y trouve simultanément le terme d'un réseau à $\mathrm{N}$ traits dont la différence de marche entre deux traits consécutifs est e $(n-1)$ et le terme d'un prisme dont l'épaisseur à la base est $\mathrm{Ne}$. Le deuxième terme est d'ailleurs beaucoup plus petit que le premier ( 10 à 100 fois), mais contrairement à ce que laisse penser l'expression algébrique, la résolution du prisme s'ajoute à celle du réseau, parce que $\partial \mathrm{n} / \partial \lambda$ est négatif.

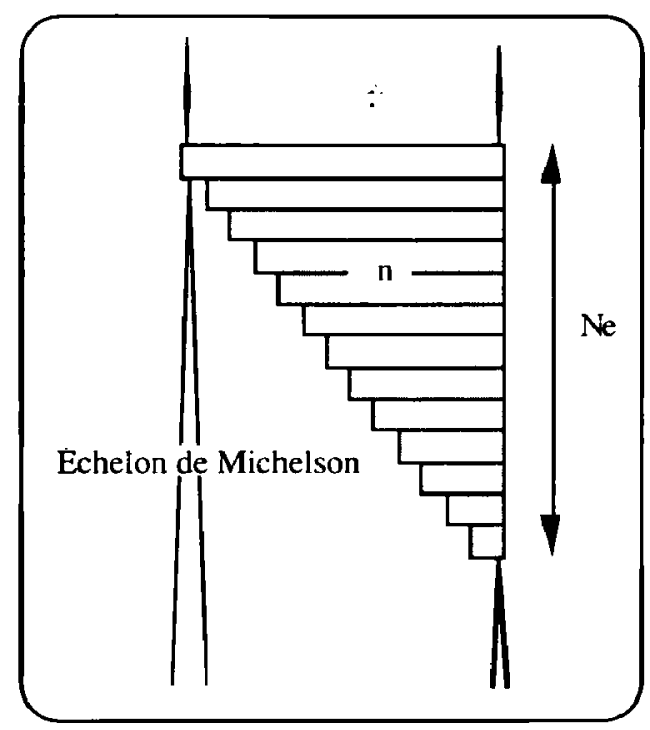

Figure 8.3 : Pouvoir de résolution théorique d'un prisme et d'un réseau associés, l'échelon de Michelson.

\subsection{Conclusion.}

Nous voyons d'après cette expression du pouvoir de résolution théorique que cette grandeur n'est pas la conséquence d'insuffisances instrumentales, mais bien celle de la nature mềme des ondes électromagnétiques. La puissance des systèmes interférentiels vient de ce qu'ils permettent d'agir directement sur le paramètre essentiel qui nous permet d'atteindre la phase des ondes optiques, à savoir la différence de marche $\mathrm{D}$ entre les rayons qui interfèrent. Si un jour nous avons à notre disposition des récepteurs suffisamment rapides et des chaînes de traitement du signal possédant les mêmes qualités, nous pourrons espérer faire de la mesure de fréquences optiques par comptage de périodes, et la formule deviendra

$$
R_{0}=\mathbf{f} . \mathbf{T}
$$

ce qui nous permettrait dans le domaine visible d'atteindre la limite d'exactitude de l'étalon de fréquence en une durée de $0,02 \mathrm{~s}$ et avec un instrument dont l'encombrement devrait être fondamentalement très réduit pour que les retards de transmission entre les différents organes ne perturbent pas le traitement de signal. Il se présentera alors une autre limitation fondamentale donnée par un nombre minimal de photons à détecter pendant la durée de la mesure, pour que le rapport signal sur bruit soit suffisant et puisse garantir la sécurité du comptage. La résolution de ces dispositifs spectrométriques du futur sera alors directement liée à la quantité d'information délivrée par la source. La technique de spectrométrie 
hétérodyne qui consiste à mesurer la fréquence de battement $f=v-v_{0}$ avec une source de référence dont la fréquence connue $v_{0}$ permet indirectement d'atteindre cette limite. Si la fréquence $v_{0}$ est supposée connue sans incertitude :

$$
R_{0}=v / \delta v=v / \delta\left(v-v_{0}\right)=v_{0} \cdot T
$$

La spectrométrie optique est donc une science où l'expérience et la technique jouent un rôle aussi important que la théorie et la connaissance de la nature des ondes électromagnétiques. Depuis l'analyse et la synthèse de la lumière blanche faite par Newton jusqu'à l'interprétation du spectre du corps noir par Max Planck et à l'interprétation du spectre de l'atome d'hydrogène par Niels Bohr, la spectrométrie optique a toujours été un moteur des progrès de la connaissance scientifique. Ce qui est devenu aujourd'hui une technique industrielle courante a été pour nous l'occasion de comprendre quelques propriétés fondamentales du champ électromagnétique, et parfois d'aborder des techniques dont les retombées débordent largement les limites de la spectrométrie optique, comme les cavités résonnantes de Fabry et Perot ou la spectrométrie par transformation de Fourier.

\section{Bibliographie}

[1] Bruhat G., Kastler A., Optique (Masson, Paris, 1992).

[2] Perez J.Ph., Optique géométrique et ondulatoire, polarisation, 3ème édition (Masson, Paris, 1990).

[3] Bousquet P., Spectrométrie Instrumentale (Dunod, Paris, 1967).

[4] Connes P., " How light is analysed ", Scientific American, septembre 1968.

[5] Jacquinot P., Girard A., Spectroscopie optique, La Recherche, 1, (1970).

[6] Jacquinot P., Girard A., Principles in experimental methods in spectroscopy, Advanced Optical Techniques (North Holland Publishing Co, Amsterdam, 1967).

[7] D'Odorico S., Instrumentation for the ESO Very Large Telescope, J. Optics, 22, 2, 1991.

[8] Bouchareine P., Techniques de l'Ingénieur, Mesures et Contrôles, Spectrométrie optique, Paris, 1994.

[9] Bouchareine P., Spectromètres et spectrographes optiques, Encyclopaedia Universalis, Paris, 1995. 